https://doi.org/10.11646/zoosymposia.17.1.14

http://zoobank.org/urn:lsid:zoobank.org:pub:228C337D-E5F8-4086-9C4A-3EB5E2BE5243

\title{
A catalog and assessment of Prof. Hideji Yamaguchi's slide collection of branchiobdellidans (Annelida: Clitellata) with the identification of syntypes
}

\author{
AKIFUMI OHTAKA ${ }^{1}$, STUART R. GELDER ${ }^{2} \&$ SUSAN M. PETERSON $^{2}$ \\ ${ }^{1}$ Faculty of Education, Hirosaki University, Hirosaki 036-8560, Japan. \\ ${ }^{2}$ Department of Science, University of Maine at Presque Isle, Maine 04769, USA. \\ Email: ohtaka@hirosaki-u.ac.jp
}

\begin{abstract}
The branchiobdellidan slide collection of Professor Hideji Yamaguchi was rediscovered in 1995; however, all of the written records associated with it appear to have been lost. The slide collection has been examined, cataloged and deposited in the Invertebrate Collection of the Hokkaido University Museum, Sapporo, Japan (ICHAM). The collection was found to contain whole mounted specimens of all 17 species described by Prof. Yamaguchi, together with specimens of three other Oriental species described after his death. In addition, mounted specimens of some of these species were designated syntypes. Many sectioned and stained specimens were also cataloged, but those were not identified to species at this time.
\end{abstract}

Key Words: Annelida, Clitellata, Branchiobdellida, taxonomy, catalog, East Asian distribution

\section{Introduction}

Branchiobdellidans, or crayfish worms, are ectosymbiotic clitellate annelids that live primarily on freshwater Holarctic astaciodian crayfishes. Based on Bănărescu's (1990) zoogeographic organization, they have an endemic, disjunct distribution in the East Asian, Euro-Mediterranean, and eastern and western North American regions (Gelder 1999; Gelder \& Williams 2016). The order Branchiobdellida Holt, 1965 is monophyletic (Williams et al. 2013) consisting of one family, Branchiobdellidae Grube, 1851 which is divided into five subfamilies (Brinkhurst \& Gelder 2001) with about 150 species in 22 genera (Gelder 1996, 2011).

A detailed historical review of branchiobdellidans in Eastern Asia was given in Gelder \& Ohtaka (2002) with subsequent studies being conducted by Gelder (2010), Niwa et al. (2005), Fujita et al. (2010), Ohtaka (2010), Ohtaka \& Chen (2010), Ohtaka et al. (2012), Ohtaka \& Gelder (2015), Ahn \& Min (2016), Tanaka et al. (2016) and Kitayabu et al. (2016). Early studies of East Asian worms were based on poorly preserved specimens (Pierantoni 1912); however, Professor Hideji Yamaguchi (1932a, b, c, 1933, 1934) ensured that his material was free from such artifacts, thus resulting in 17 new species and some redescriptions that, together, formed the first comprehensive investigation of branchiobdellidans in East Asia. Unfortunately, he did not designate any type series for these species, nor state in which institution his material had been deposited. After Yamaguchi's death in 1970, it was believed that both his slides and his fluid-preserved specimen collection of branchiobdellidans had been lost and probably destroyed, until one of us (A.O.) found the slide collection in 1995 (Gelder \& Ohtaka 2002).

A preliminary study of the slides revealed they consisted of whole mount and sectioned specimens with some having annotation. Unfortunately, no catalogs or recorded information accompanied the collection. Although many of the whole specimens could be identified to species, others labeled "unidentified" may still be named following further study. Yamaguchi's sections were of a high standard, but given the limited conditions at the time and without labels, these specimens had to be set aside for future study. Consequently, 
the preliminary report (Gelder \& Ohtaka 2002) contained a list of species thus far identified by the authors and a summary of other details then available. Additional details obtained since 2002 are included in this present paper: the collection—and particularly the sectioned material-remains to be fully investigated. To this end, the details included in our Table 1 were designed to assist future researchers by ensuring all the information currently available is not lost.

TABLE 1. Microscope slides of branchiobdellidans in the Prof. H. Yamaguchi collection. Each slide is arranged by its original box/slide number, assigned museum number, original slide markings, specimen(s) status, species identification and type status followed by the Japanese common name.

\begin{tabular}{|c|c|c|c|c|c|c|}
\hline $\begin{array}{l}\text { Box / } \\
\text { Slide } \\
\text { No. }\end{array}$ & Catalog No. & Slide Markings & $\begin{array}{l}\text { Specimen(s) } \\
\text { Status }\end{array}$ & Species Ident. & Type Status & $\begin{array}{l}\text { Japanese } \\
\text { common name }\end{array}$ \\
\hline 1.2 & ICHUM-1398 & c.d.l. 3 & sect. & - & & - \\
\hline 1.4 & ICHUM-1399 & E. 16 / Sp.E / D.1 & WM & $\begin{array}{l}\text { Cirrodrilus } \\
\text { nipponicus } \\
\text { (Yamaguchi, 1932) }\end{array}$ & syntype & $\begin{array}{l}\text { Nippon- } \\
\text { zariganimimizu }\end{array}$ \\
\hline 1.6 & ICHUM-1400 & A. $10 /$ A. / 107 & WM 1 & $\begin{array}{l}\text { Cirrodrilus cirratus } \\
\text { Pierantoni, } 1905\end{array}$ & non-type & $\begin{array}{l}\text { Kamuri- } \\
\text { zariganimimizu }\end{array}$ \\
\hline$"$ & ICHUM-1401 & $"$ & WM 2 & $\begin{array}{l}\text { Cirrodrilus cirratus } \\
\text { Pierantoni, } 1905\end{array}$ & non-type & $\begin{array}{l}\text { Kamuri- } \\
\text { zariganimimizu }\end{array}$ \\
\hline $1.8 *$ & ICHUM-1402 & H. $4 * *$ & WM & $\begin{array}{l}\text { Cirrodrilus makinoi } \\
\text { (Yamaguchi, 1934) }\end{array}$ & syntype & $\begin{array}{l}\text { Hime- } \\
\text { zariganimimizu }\end{array}$ \\
\hline 1.9 & ICHUM-1403 & H. 5 / c.m.b. & sect. & - & & - \\
\hline 1. 14 & ICHUM-1404 & H. $3 * *$ & WM & $\begin{array}{l}\text { Cirrodrilus makinoi } \\
\text { (Yamaguchi, 1934) }\end{array}$ & syntype & $\begin{array}{l}\text { Hime- } \\
\text { zariganimimizu }\end{array}$ \\
\hline 1. 17 & ICHUM-1405 & H. $-14 * *$ & WM 1 & $\begin{array}{l}\text { Cirrodrilus makinoi } \\
\text { (Yamaguchi, 1934) }\end{array}$ & syntype & $\begin{array}{l}\text { Hime- } \\
\text { zariganimimizu }\end{array}$ \\
\hline$"$ & ICHUM-1406 & $"$ & WM 2 & $\begin{array}{l}\text { Cirrodrilus makinoi } \\
\text { (Yamaguchi, 1934) }\end{array}$ & syntype & $\begin{array}{l}\text { Hime- } \\
\text { zariganimimizu }\end{array}$ \\
\hline 1. 19 & ICHUM-1407 & E. $11 / 2 /$ E. 2 & sect. & - & & - \\
\hline $1.20 *$ & ICHUM-1408 & 20 & WM 1 & $\begin{array}{l}\text { Cirrodrilus makinoi } \\
\text { (Yamaguchi, 1934) }\end{array}$ & syntype & $\begin{array}{l}\text { Hime- } \\
\text { zariganimimizu }\end{array}$ \\
\hline$"$ & ICHUM-1409 & $"$ & WM 2 & $\begin{array}{l}\text { Cirrodrilus makinoi } \\
\text { (Yamaguchi, 1934) }\end{array}$ & syntype & $\begin{array}{l}\text { Hime- } \\
\text { zariganimimizu }\end{array}$ \\
\hline 1.23 & ICHUM-1410 & Sp. E. 8 - 2 & sect. & - & & - \\
\hline 1.25 & ICHUM-1411 & Sp. E. 9 - 2 & sect. & - & & - \\
\hline 1.26 & ICHUM-1412 & Sp. E. 9 - 1 & sect. & - & & - \\
\hline 1.27 & ICHUM-1413 & Sp. E. 8 - 4 & sect. & - & & - \\
\hline 1. 28 & ICHUM-1414 & Sp. E. 8 - 3 & sect. & - & & - \\
\hline 1. 29 & ICHUM-1415 & Sp. E. 8 - 1 & sect. & - & & - \\
\hline 1.30 & ICHUM-1416 & Sp. E. 7 - 2 & sect. & - & & - \\
\hline
\end{tabular}


TABLE 1. (Continued)

\begin{tabular}{|c|c|c|c|c|c|c|}
\hline $\begin{array}{l}\text { Box / } \\
\text { Slide } \\
\text { No. }\end{array}$ & Catalog No. & Slide Markings & $\begin{array}{l}\text { Specimen(s) } \\
\text { Status }\end{array}$ & Species Ident. & Type Status & $\begin{array}{l}\text { Japanese } \\
\text { common name }\end{array}$ \\
\hline 1.31 & ICHUM-1417 & Sp. E. 7 - 1 & sect. & - & & - \\
\hline 1. 32 & ICHUM-1418 & Sp. E. $6 /($ APF?) $* *$ & sect. & - & & - \\
\hline 1.33 & ICHUM-1419 & E. 5 & sect. & - & & - \\
\hline 1. 34 & ICHUM-1420 & E. 4 & sect. & - & & - \\
\hline 1.35 & ICHUM-1421 & Sp. E 3 & sect. & - & & - \\
\hline 1.36 & ICHUM-1422 & E. 2 & sect. & - & & - \\
\hline 1.37 & ICHUM-1423 & E. 1 - 2 & sect. & - & & - \\
\hline 1. 38 & ICHUM-1424 & Sp E 1 & sect. & - & & - \\
\hline 1.39 & ICHUM-1425 & c.m.b. 19 / 19 & sect. & - & & - \\
\hline 1. 40 & ICHUM-1426 & c.m.b 20 / 1 / 12-1 & sect. & - & & - \\
\hline 1. 41 & ICHUM-1427 & c.m.b.-20 / 2 / 12-2 & sect. & - & & - \\
\hline 1.42 & ICHUM-1428 & c.m.b 20 - 3 / 12-3 & sect. & - & & - \\
\hline 1.43 & ICHUM-1429 & c.m.b $21 / 10$ & sect. & - & & - \\
\hline 1.44 & ICHUM-1430 & K. 6 - 2 & sect. & - & & - \\
\hline $1.46 *$ & ICHUM-1431 & $\mathrm{K} 6 / 6$ & WM & $\begin{array}{l}\text { Hidejiodrilus } \\
\text { koreanus (Pierantoni, } \\
1912 \text { ) }\end{array}$ & non-type & $\begin{array}{l}\text { Yotsuyubi- } \\
\text { zariganimimizu }\end{array}$ \\
\hline 1.47 & ICHUM-1432 & K. $6 / 5$ & sect. & - & & - \\
\hline 1. 48 & ICHUM-1433 & K. $6 / 4$ & sect. & - & & - \\
\hline 1. 49 & ICHUM-1434 & K. $6 / 3$ - 2 & sect. & - & & - \\
\hline 1.50 & ICHUM-1435 & K. $6 / 3$ - 1 & sect. & - & & - \\
\hline 1.51 & ICHUM-1436 & $\mathrm{F} 3 * *$ & WM & $\begin{array}{l}\text { Cirrodrilus uchidai } \\
\text { (Yamaguchi, 1932)? }\end{array}$ & syntype ? & $\begin{array}{l}\text { Uchida- } \\
\text { zariganimimizu }\end{array}$ \\
\hline 1. $52 *$ & ICHUM-1437 & $\mathrm{F} 4 * *$ & $\mathrm{WM}$ & $\begin{array}{l}\text { Cirrodrilus uchidai } \\
\text { (Yamaguchi, 1932)? }\end{array}$ & syntype? & $\begin{array}{l}\text { Uchida- } \\
\text { zariganimimizu }\end{array}$ \\
\hline 1.54 & ICHUM-1438 & $\mathrm{F} 6 * *$ & WM & $\begin{array}{l}\text { Cirrodrilus uchidai } \\
\text { (Yamaguchi, 1932)? }\end{array}$ & syntype? & $\begin{array}{l}\text { Uchida- } \\
\text { zariganimimizu } \\
?\end{array}$ \\
\hline 1.56 & ICHUM-1439 & Sp. E / D.3 / E 15 & WM & $\begin{array}{l}\text { Cirrodrilus } \\
\text { nipponicus } \\
\text { (Yamaguchi, 1932) }\end{array}$ & syntype ? & $\begin{array}{l}\text { Nippon- } \\
\text { zariganimimizu }\end{array}$ \\
\hline 1.58 & ICHUM-1440 & H. 9 - 2 / H. K. 1 - 2 & sect. & - & & - \\
\hline
\end{tabular}


TABLE 1. (Continued)

\begin{tabular}{|c|c|c|c|c|c|c|}
\hline $\begin{array}{l}\text { Box / } \\
\text { Slide } \\
\text { No. }\end{array}$ & Catalog No. & Slide Markings & $\begin{array}{l}\text { Specimen(s) } \\
\text { Status }\end{array}$ & Species Ident. & Type Status & $\begin{array}{l}\text { Japanese } \\
\text { common name }\end{array}$ \\
\hline 1.59 & ICHUM-1441 & E. 19 & WM & $\begin{array}{l}\text { Cirrodrilus } \\
\text { nipponicus } \\
\text { (Yamaguchi, 1932) }\end{array}$ & syntype ? & $\begin{array}{l}\text { Nippon- } \\
\text { zariganimimizu }\end{array}$ \\
\hline 1.60 & ICHUM-1442 & c.d $1 . / 1 /$ c.d 1 & sect. & - & & - \\
\hline 1. 61 & ICHUM-1443 & c.s. - 1 & sect. & - & & - \\
\hline 1.62 & ICHUM-1444 & c.s. - 2 & sect. & - & & - \\
\hline 1.63 & ICHUM-1445 & c.s. - 3 & sect. & - & & - \\
\hline 1.64 & ICHUM-1446 & c.s. - 18 & sect. & - & & - \\
\hline 1. 65 & ICHUM-1447 & Sp. c.s. 4 - 2 & sect. & - & & - \\
\hline 1.66 & ICHUM-1448 & Sp. c.s. 6 - 1 & sect. & - & & - \\
\hline 1.67 & ICHUM-1449 & Sp. c.s. $6-2 * *$ & sect. & - & & - \\
\hline 1. 68 & ICHUM-1450 & Sp. c.s. 7 & sect. & - & & - \\
\hline 1.69 & ICHUM-1451 & c.s. -15 & sect. & - & & - \\
\hline 1.70 & ICHUM-1452 & c.s. 9 & sect. & - & & - \\
\hline 1.71 & ICHUM-1453 & c.s. -10 & sect. & - & & - \\
\hline 1.72 & ICHUM-1454 & c.s. - 11 & sect. & - & & - \\
\hline 1.73 & ICHUM-1455 & c.s. -12 & sect. & - & & - \\
\hline 1.74 & ICHUM-1456 & c.s. -14 & sect. & - & & - \\
\hline 1.75 & ICHUM-1457 & c.s. - 13 & sect. & - & & - \\
\hline 1.76 & ICHUM-1458 & Sp. c.s. - 5 & sect. & - & & - \\
\hline 1.77 & ICHUM-1459 & c.s. - 17 & sect. & - & & - \\
\hline 1.78 & ICHUM-1460 & E. 10 & sect. & - & & - \\
\hline 1.79 & ICHUM-1461 & H. $20-2$ & sect. & - & & - \\
\hline 1.83 & ICHUM-1462 & H $9-1 /$ H $21-1$ & sect. & - & & - \\
\hline 1.86 & ICHUM-1463 & E 21 & WM & $\begin{array}{l}\text { Cirrodrilus } \\
\text { nipponicus } \\
\text { (Yamaguchi, 1932) }\end{array}$ & syntype ? & $\begin{array}{l}\text { Nippon- } \\
\text { zariganimimizu }\end{array}$ \\
\hline $1.88 *$ & ICHUM-1464 & E 20 & WM & $\begin{array}{l}\text { Cirrodrilus } \\
\text { nipponicus } \\
\text { (Yamaguchi, 1932) }\end{array}$ & syntype? & $\begin{array}{l}\text { Nippon- } \\
\text { zariganimimizu }\end{array}$ \\
\hline 1.92 & ICHUM-1465 & H. $10-1$ & sect. & - & & - \\
\hline
\end{tabular}

...Continued on the next page 
TABLE 1. (Continued)

\begin{tabular}{|c|c|c|c|c|c|c|}
\hline $\begin{array}{l}\text { Box / } \\
\text { Slide } \\
\text { No. }\end{array}$ & Catalog No. & Slide Markings & $\begin{array}{l}\text { Specimen(s) } \\
\text { Status }\end{array}$ & Species Ident. & Type Status & $\begin{array}{l}\text { Japanese } \\
\text { common name }\end{array}$ \\
\hline 1.99 & ICHUM-1466 & K $6 / 7$ & WM & $\begin{array}{l}\text { Hidejiodrilus } \\
\text { koreanus (Pierantoni, } \\
\text { 1912) }\end{array}$ & non-type & $\begin{array}{l}\text { Yotsuyubi- } \\
\text { zariganimimizu }\end{array}$ \\
\hline 2.4 & ICHUM-1348 & 4 & WM & $\begin{array}{l}\text { Cambarincola } \\
\text { okadai Yamaguchi, } \\
1933\end{array}$ & $\begin{array}{l}\text { paralectoty } \\
\text { pe }\end{array}$ & Yadorimimizu \\
\hline 2.6 & ICHUM-1467 & am $2-10$ & sect. & - & & - \\
\hline 2.8 & ICHUM-1468 & A 8 & sect. & - & & - \\
\hline 2.9 & $\begin{array}{l}\text { USNM } \\
186575\end{array}$ & 9 & WM & $\begin{array}{l}\text { Cambarincola } \\
\text { okadai Yamaguchi, } \\
1933\end{array}$ & syntype & Yadorimimizu \\
\hline 2. $10 *$ & ICHUM-1349 & 10 & WM & $\begin{array}{l}\text { Cambarincola } \\
\text { okadai Yamaguchi, } \\
1933\end{array}$ & $\begin{array}{l}\text { paralectoty } \\
\text { pe }\end{array}$ & Yadorimimizu \\
\hline 2. 12 & ICHUM-1469 & D. $11-1$ & sect. & - & & - \\
\hline 2. 13 & ICHUM-1470 & $\mathrm{D} 12 / 1 / 4$ & sect. & - & & - \\
\hline 2. 14 & ICHUM-1471 & D. $10-2$ & sect. & - & & - \\
\hline $2.15 *$ & ICHUM-1472 & c.s $33 * *$ & WM 1 & $\begin{array}{l}\text { Cirrodrilus inukaii } \\
\text { (Yamaguchi, 1934) }\end{array}$ & syntype & $\begin{array}{l}\text { Inukai- } \\
\text { zariganimimizu }\end{array}$ \\
\hline " & ICHUM-1473 & $"$ & WM 2 & $\begin{array}{l}\text { Cirrodrilus inukaii } \\
\text { (Yamaguchi, 1934) }\end{array}$ & syntype & $\begin{array}{l}\text { Inukai- } \\
\text { zariganimimizu }\end{array}$ \\
\hline$"$ & ICHUM-1474 & $"$ & WM 3 & $\begin{array}{l}\text { Cirrodrilus inukaii } \\
\text { (Yamaguchi, 1934) }\end{array}$ & syntype & $\begin{array}{l}\text { Inukai- } \\
\text { zariganimimizu }\end{array}$ \\
\hline " & ICHUM-1475 & $"$ & WM 4 & $\begin{array}{l}\text { Cirrodrilus inukaii } \\
\text { (Yamaguchi, 1934) }\end{array}$ & syntype & $\begin{array}{l}\text { Inukai- } \\
\text { zariganimimizu }\end{array}$ \\
\hline 2. 16 & ICHUM-1476 & D. $10-1$ & sect. & - & & - \\
\hline 2. 18 & ICHUM-1477 & $18 / 2-9$ & sect. & - & & - \\
\hline 2. $20 *$ & ICHUM-1347 & 20 & WM & $\begin{array}{l}\text { Cambarincola } \\
\text { okadai Yamaguchi, } \\
1933\end{array}$ & lectotype & Yadorimimizu \\
\hline 2. 21 & ICHUM-1478 & A. 7 / H & sect. & - & & - \\
\hline 2. 22 & ICHUM-1479 & D. $9-2 / 3$ & sect. & - & & - \\
\hline 2. 23 & ICHUM-1480 & c.m.b. 7 - 2 & sect. & - & & - \\
\hline $2.26 *$ & ICHUM-1481 & 26 & WM & $\begin{array}{l}\text { Cirrodrilus } \\
\text { nipponicus } \\
\text { (Yamaguchi, 1932) }\end{array}$ & syntype? & $\begin{array}{l}\text { Nippon- } \\
\text { zariganimimizu }\end{array}$ \\
\hline
\end{tabular}

...Continued on the next page 
TABLE 1. (Continued)

\begin{tabular}{|c|c|c|c|c|c|c|}
\hline $\begin{array}{l}\text { Box / } \\
\text { Slide } \\
\text { No. }\end{array}$ & Catalog No. & Slide Markings & $\begin{array}{l}\text { Specimen(s) } \\
\text { Status }\end{array}$ & Species Ident. & Type Status & $\begin{array}{l}\text { Japanese } \\
\text { common name }\end{array}$ \\
\hline 2.29 & ICHUM-1482 & $5-2$ & sect. & - & & - \\
\hline 2.32 & ICHUM-1483 & A $6-2$ & sect. & - & & - \\
\hline 2. 34 & ICHUM-1484 & III / c.d.s 16 & sect. & - & & - \\
\hline 2.35 & ICHUM-1485 & II / c.d.s 11 & sect. & - & & - \\
\hline 2.36 & ICHUM-1486 & I / c.d.s 16 & sect. & - & & - \\
\hline 2. 40 & ICHUM-1487 & $\begin{array}{l}\text { c. d. s. / (?) / c d s / } \\
14\end{array}$ & sect. & - & & - \\
\hline 2. 41 & ICHUM-1488 & 8 & sect. & - & & - \\
\hline 2.43 & ICHUM-1489 & 43 & WM & $\begin{array}{l}\text { Cirrodrilus cirratus } \\
\text { Pierantoni, } 1905\end{array}$ & non-type & $\begin{array}{l}\text { Kamuri- } \\
\text { zariganimimizu }\end{array}$ \\
\hline 2. 44 & ICHUM-1490 & $10 / \mathrm{D} 5$ & sect. & - & & - \\
\hline 2. 46 & ICHUM-1491 & 8 & sect. & - & & - \\
\hline 2. 48 & ICHUM-1492 & $\begin{array}{l}\text { D / } 2.2-3 / \text { D. } 8 \text { - } \\
3\end{array}$ & sect. & - & & - \\
\hline 2. 49 & ICHUM-1493 & 49 & WM & unidentified & & - \\
\hline 2.50 & ICHUM-1494 & Sp.cmb / D.1 & WM 1 & $\begin{array}{l}\text { Cirrodrilus } \\
\text { homodontus } \\
\text { (Yamaguchi, 1932) }\end{array}$ & syntype? & $\begin{array}{l}\text { Hoso- } \\
\text { zariganimimizu }\end{array}$ \\
\hline " & ICHUM-1495 & $"$ & WM 2 & unidentified & & - \\
\hline " & ICHUM-1496 & $"$ & WM 3 & unidentified & & - \\
\hline 2.54 & ICHUM-1497 & $54 * *$ & WM & $\begin{array}{l}\text { Cirrodrilus uchidai } \\
\text { (Yamaguchi, 1932)? }\end{array}$ & syntype? & $\begin{array}{l}\text { Uchida- } \\
\text { zariganimimizu }\end{array}$ \\
\hline 2.55 & ICHUM-1498 & $3 / \mathrm{D} 5$ & sect. & - & & - \\
\hline 2.56 & ICHUM-1499 & c.m.b. 2 & sect. & - & & - \\
\hline 2.57 & ICHUM-1500 & D. 9 - 1 & sect. & - & & - \\
\hline 2.58 & ICHUM-1501 & D. $6-6$ & sect. & - & & - \\
\hline 2.59 & ICHUM-1502 & D. $6-5$ & sect. & - & & - \\
\hline 2. $60 *$ & ICHUM-1503 & 59 & WM & $\begin{array}{l}\text { Cirrodrilus uchidai } \\
\text { (Yamaguchi, 1932)? }\end{array}$ & syntype ? & $\begin{array}{l}\text { Uchida- } \\
\text { zariganimimizu }\end{array}$ \\
\hline 2. 61 & ICHUM-1504 & $61 * *$ & - & - & & - \\
\hline 2. 62 & ICHUM-1505 & c.m.b. / 62 & sect. & - & & - \\
\hline 2. 63 & ICHUM-1506 & $\mathrm{F} 2 * *$ & sect. & - & & - \\
\hline
\end{tabular}

...Continued on the next page 
TABLE 1. (Continued)

\begin{tabular}{|c|c|c|c|c|c|c|}
\hline $\begin{array}{l}\text { Box / } \\
\text { Slide } \\
\text { No. }\end{array}$ & Catalog No. & Slide Markings & $\begin{array}{l}\text { Specimen(s) } \\
\text { Status }\end{array}$ & Species Ident. & Type Status & $\begin{array}{l}\text { Japanese } \\
\text { common name }\end{array}$ \\
\hline 2.64 & ICHUM-1507 & D. 9 - 3 & sect. & - & & - \\
\hline 2.65 & ICHUM-1508 & D. $12 / 2 / 3$ & sect. & - & & - \\
\hline 2.66 & ICHUM-1509 & D. 11 - 2 / 2 & sect. & - & & - \\
\hline 2.67 & ICHUM-1510 & D. 11.4 & sect. & - & & - \\
\hline 2.68 & ICHUM-1511 & D. 6 - 4 & sect. & - & & - \\
\hline 2. 69 & ICHUM-1512 & D. $12 / 3 / 2$ & sect. & - & & - \\
\hline 2.70 & ICHUM-1513 & $7-7$ & sect. & - & & - \\
\hline 2.71 & ICHUM-1514 & D. 6 - 7 & sect. & - & & - \\
\hline 2.72 & ICHUM-1515 & $7-1$ & sect. & - & & - \\
\hline 2.74 & ICHUM-1516 & D. 12 / 4 / D & sect. & - & & - \\
\hline 2.77 & ICHUM-1517 & $1-4$ & sect. & - & & - \\
\hline 2.78 & ICHUM-1518 & $\mathrm{am} / 1-2$ & sect. & - & & - \\
\hline 2.80 & ICHUM-1519 & $2-7$ & sect. & - & & - \\
\hline 2.82 & ICHUM-1520 & am $2-1$ & sect. & destroyed & & - \\
\hline 2.84 & ICHUM-1521 & $2-4$ & sect. & - & & - \\
\hline 2.86 & ICHUM-1522 & $\mathrm{am} / 2-2$ & sect. & - & & - \\
\hline 2.87 & ICHUM-1523 & $2-3$ & sect. & - & & - \\
\hline 2.89 & ICHUM-1524 & $\mathrm{am} / 2-5$ & sect. & - & & - \\
\hline 2.90 & ICHUM-1525 & $2-6$ & sect. & - & & - \\
\hline 2.91 & ICHUM-1526 & $2-8$ & sect. & - & & - \\
\hline 2.92 & ICHUM-1527 & a.m 7 - 5 & sect. & - & & - \\
\hline 2.93 & ICHUM-1528 & $1-3$ & sect. & - & & - \\
\hline 2.95 & ICHUM-1529 & D. 6 - 2 & sect. & - & & - \\
\hline 2.96 & ICHUM-1530 & D. 6 - 1 & sect. & - & & - \\
\hline 2.97 & ICHUM-1531 & ext & sect. & destroyed & & - \\
\hline 2.98 & ICHUM-1532 & $13 / D-5$ & sect. & - & & - \\
\hline 2.99 & ICHUM-1533 & D. $6-3 \mathrm{~A}$ & sect. & - & & - \\
\hline 2. 100 & ICHUM-1534 & D. 6 - 3 B & sect. & - & & - \\
\hline
\end{tabular}


TABLE 1. (Continued)

\begin{tabular}{|c|c|c|c|c|c|c|}
\hline $\begin{array}{l}\text { Box / } \\
\text { Slide } \\
\text { No. }\end{array}$ & Catalog No. & Slide Markings & $\begin{array}{l}\text { Specimen(s) } \\
\text { Status }\end{array}$ & Species Ident. & Type Status & $\begin{array}{l}\text { Japanese } \\
\text { common name }\end{array}$ \\
\hline 3.1 & ICHUM-1535 & D. $5 / 2$ & sect. & - & & - \\
\hline 3.2 & ICHUM-1536 & D $4 / 7$ & sect. & - & & - \\
\hline 3.3 & ICHUM-1537 & D. 5 / 1 & sect. & - & & - \\
\hline 3.4 & ICHUM-1538 & D $4 / 6$ & sect. & - & & - \\
\hline 3.5 & ICHUM-1539 & D $4 / 8$ & sect. & - & & - \\
\hline 3.6 & ICHUM-1540 & D $4 / 5$ & sect. & - & & - \\
\hline 3.7 & ICHUM-1541 & D $4 / 4$ & sect. & - & & - \\
\hline 3.8 & ICHUM-1542 & D $4 / 3$ & sect. & - & & - \\
\hline 3.9 & ICHUM-1543 & D $4 / 2$ & sect. & - & & - \\
\hline 3. 10 & ICHUM-1544 & $10 * *$ & sect. & - & & - \\
\hline 3.11 & ICHUM-1545 & D $3 / 35$ & sect. & - & & - \\
\hline 3. 12 & ICHUM-1546 & D $3 / 34$ & sect. & - & & - \\
\hline 3.13 & ICHUM-1547 & D. 3 / 33 & sect. & - & & - \\
\hline 3.14 & ICHUM-1548 & D $3 / 32$ & sect. & - & & - \\
\hline 3. 15 & ICHUM-1549 & D $3 / 31$ & sect. & - & & - \\
\hline 3. 16 & ICHUM-1550 & D. 3 / 30 & sect. & - & & - \\
\hline 3. 17 & ICHUM-1551 & D. 3 / 29 & sect. & - & & - \\
\hline 3. 18 & ICHUM-1552 & D. $3 / 28$ & sect. & - & & - \\
\hline 3. 19 & ICHUM-1553 & D. $3 / 27$ & sect. & - & & - \\
\hline $3.20 *$ & ICHUM-1554 & D. 14 & WM & $\begin{array}{l}\text { Cirrodrilus } \\
\text { sapporensis } \\
\text { (Pierantoni, 1906) }\end{array}$ & non-type & Zariganimimizu \\
\hline 3. 21 & ICHUM-1555 & D. 15 & WM 1 & $\begin{array}{l}\text { Cirrodrilus } \\
\text { sapporensis } \\
\text { (Pierantoni, 1906) }\end{array}$ & non-type & Zariganimimizu \\
\hline$"$ & ICHUM-1556 & $"$ & WM 2 & $\begin{array}{l}\text { Cirrodrilus } \\
\text { sapporensis } \\
\text { (Pierantoni, 1906) }\end{array}$ & non-type & Zariganimimizu \\
\hline 3.22 & ICHUM-1557 & D. 16 & WM & $\begin{array}{l}\text { Cirrodrilus } \\
\text { sapporensis } \\
\text { (Pierantoni, 1906) }\end{array}$ & non-type & Zariganimimizu \\
\hline 3.23 & ICHUM-1558 & D. 17 & WM & unidentified & & - \\
\hline
\end{tabular}


TABLE 1. (Continued)

\begin{tabular}{|c|c|c|c|c|c|c|}
\hline $\begin{array}{l}\text { Box / } \\
\text { Slide } \\
\text { No. }\end{array}$ & Catalog No. & Slide Markings & $\begin{array}{l}\text { Specimen(s) } \\
\text { Status }\end{array}$ & Species Ident. & Type Status & $\begin{array}{l}\text { Japanese } \\
\text { common name }\end{array}$ \\
\hline 3.25 & ICHUM-1559 & D. 19 & $\mathrm{WM}$ & $\begin{array}{l}\text { Cirrodrilus } \\
\text { sapporensis } \\
\text { (Pierantoni, 1906) }\end{array}$ & non-type & Zariganimimizu \\
\hline $3.26 *$ & ICHUM-1560 & D 21 & WM & destroyed & & - \\
\hline $3.28 *$ & ICHUM-1561 & D. 22 & $\mathrm{WM}$ & $\begin{array}{l}\text { Cirrodrilus } \\
\text { sapporensis } \\
\text { (Pierantoni, 1906) }\end{array}$ & non-type & Zariganimimizu \\
\hline $3.29 *$ & ICHUM-1562 & D. 23 & WM 1 & $\begin{array}{l}\text { Cirrodrilus } \\
\text { sapporensis } \\
\text { (Pierantoni, 1906) }\end{array}$ & non-type & Zariganimimizu \\
\hline$"$ & ICHUM-1563 & $"$ & WM 2 & $\begin{array}{l}\text { Cirrodrilus } \\
\text { sapporensis } \\
\text { (Pierantoni, 1906) }\end{array}$ & non-type & Zariganimimizu \\
\hline $3.32 *$ & ICHUM-1564 & D. 24 & WM & $\begin{array}{l}\text { Cirrodrilus } \\
\text { sapporensis } \\
\text { (Pierantoni, 1906) }\end{array}$ & non-type & Zariganimimizu \\
\hline 3.34 & ICHUM-1565 & D. 1 - 1 & sect. & - & & - \\
\hline 3.35 & ICHUM-1566 & D. 1 - 2 & sect. & - & & - \\
\hline 3.36 & ICHUM-1567 & D. 1 - 4 & sect. & - & & - \\
\hline 3.38 & ICHUM-1568 & D. 1 - 5 & sect. & - & & - \\
\hline 3.39 & ICHUM-1569 & D. 1 - 6 & sect. & - & & - \\
\hline 3. 40 & ICHUM-1570 & D. $1-7$ & sect. & - & & - \\
\hline 3.41 & ICHUM-1571 & D. - 2 - 1 & sect. & - & & - \\
\hline 3. 42 & ICHUM-1572 & D. 2 - 2 & sect. & - & & - \\
\hline 3.43 & ICHUM-1573 & D. 2 - 3 & sect. & - & & - \\
\hline 3. 44 & ICHUM-1574 & D. 2 - 4 & sect. & - & & - \\
\hline 3.45 & ICHUM-1575 & D. 2 - 5 & sect. & - & & - \\
\hline 3. 46 & ICHUM-1576 & D. 2 - 6 & sect. & - & & - \\
\hline 3. 47 & ICHUM-1577 & D. 2 - 7 & sect. & - & & - \\
\hline 3. 48 & ICHUM-1578 & D. $3-1 / 1$ & sect. & - & & - \\
\hline 3. 49 & ICHUM-1579 & D. $3-2 / 2$ & sect. & - & & - \\
\hline 3. 50 & ICHUM-1580 & D. $3-3 / 3$ & sect. & - & & - \\
\hline 3.51 & ICHUM-1581 & D. $3 / 4 / 4$ & sect. & - & & - \\
\hline
\end{tabular}


TABLE 1. (Continued)

\begin{tabular}{|c|c|c|c|c|c|c|}
\hline $\begin{array}{l}\text { Box / } \\
\text { Slide } \\
\text { No. }\end{array}$ & Catalog No. & Slide Markings & $\begin{array}{l}\text { Specimen(s) } \\
\text { Status }\end{array}$ & Species Ident. & Type Status & $\begin{array}{l}\text { Japanese } \\
\text { common name }\end{array}$ \\
\hline 3.52 & ICHUM-1582 & D. $3 / 5 / 5$ & sect. & - & & - \\
\hline 3.53 & ICHUM-1583 & D. $3 / 6 / 6$ & sect. & - & & - \\
\hline 3.54 & ICHUM-1584 & 67 & sect. & - & & - \\
\hline $3.55 *$ & ICHUM-1585 & 55 & WM 1 & $\begin{array}{l}\text { Cirrodrilus inukaii } \\
\text { (Yamaguchi, 1934) }\end{array}$ & syntype & $\begin{array}{l}\text { Inukai- } \\
\text { zariganimimizu }\end{array}$ \\
\hline$"$ & ICHUM-1586 & $"$ & WM 2 & unidentified & & - \\
\hline 3.56 & ICHUM-1587 & 56 & $\mathrm{WM}$ & unidentified & & - \\
\hline $3.57 *$ & ICHUM-1588 & 57 & WM 1 & $\begin{array}{l}\text { Cirrodrilus inukaii } \\
\text { (Yamaguchi, 1934) }\end{array}$ & syntype & $\begin{array}{l}\text { Inukai- } \\
\text { zariganimimizu }\end{array}$ \\
\hline$"$ & ICHUM-1589 & $"$ & WM 2 & $\begin{array}{l}\text { Cirrodrilus inukaii } \\
\text { (Yamaguchi, 1934) }\end{array}$ & syntype & $\begin{array}{l}\text { Inukai- } \\
\text { zariganimimizu }\end{array}$ \\
\hline 3.58 & ICHUM-1590 & 58 & WM 1 & $\begin{array}{l}\text { Cirrodrilus inukaii } \\
\text { (Yamaguchi, 1934) }\end{array}$ & syntype & $\begin{array}{l}\text { Inukai- } \\
\text { zariganimimizu }\end{array}$ \\
\hline$"$ & ICHUM-1591 & $"$ & WM 2 & $\begin{array}{l}\text { Cirrodrilus inukaii } \\
\text { (Yamaguchi, 1934) }\end{array}$ & syntype & $\begin{array}{l}\text { Inukai- } \\
\text { zariganimimizu }\end{array}$ \\
\hline 3.59 & ICHUM-1592 & 59 & WM 1 & $\begin{array}{l}\text { Cirrodrilus inukaii } \\
\text { (Yamaguchi, 1934) }\end{array}$ & syntype & $\begin{array}{l}\text { Inukai- } \\
\text { zariganimimizu }\end{array}$ \\
\hline$"$ & ICHUM-1593 & $"$ & WM 2 & $\begin{array}{l}\text { Cirrodrilus inukaii } \\
\text { (Yamaguchi, 1934) }\end{array}$ & syntype & $\begin{array}{l}\text { Inukai- } \\
\text { zariganimimizu }\end{array}$ \\
\hline 3.60 & ICHUM-1594 & 60 & WM & unidentified & & - \\
\hline 3.61 & ICHUM-1595 & 61 & WM & unidentified & & - \\
\hline 3.62 & ICHUM-1596 & D. $3 / 26 / 26$ & sect. & - & & - \\
\hline 3.63 & ICHUM-1597 & D. $3 / 25 / 25$ & sect. & - & & - \\
\hline 3.64 & ICHUM-1598 & D. $3 / 24 / 24$ & sect. & - & & - \\
\hline 3.65 & ICHUM-1599 & D. 3 / 23 / 23 & sect. & - & & - \\
\hline 3.66 & ICHUM-1600 & D. $3 / 22 / 22$ & sect. & - & & - \\
\hline 3.67 & ICHUM-1601 & D. $3 / 21 / 21$ & sect. & destroyed & & - \\
\hline 3.68 & ICHUM-1602 & D. $3 / 20 / 20$ & sect. & - & & - \\
\hline 3.69 & ICHUM-1603 & D. 3 / 19 / 19 & sect. & - & & - \\
\hline 3.70 & ICHUM-1604 & D. $3 / 18 / 18$ & sect. & - & & - \\
\hline 3.71 & ICHUM-1605 & D. 3 / 17 / 17 & sect. & - & & - \\
\hline 3.72 & ICHUM-1606 & D. $3 / 16 / 16$ & sect. & - & & - \\
\hline
\end{tabular}

...Continued on the next page 
TABLE 1. (Continued)

\begin{tabular}{|c|c|c|c|c|c|c|}
\hline $\begin{array}{l}\text { Box / } \\
\text { Slide } \\
\text { No. }\end{array}$ & Catalog No. & Slide Markings & $\begin{array}{l}\text { Specimen(s) } \\
\text { Status }\end{array}$ & Species Ident. & Type Status & $\begin{array}{l}\text { Japanese } \\
\text { common name }\end{array}$ \\
\hline 3.73 & ICHUM-1607 & D. $3 / 15 / 15$ & sect. & - & & - \\
\hline 3.74 & ICHUM-1608 & D. $3 / 14$ / 14 & sect. & - & & - \\
\hline 3.75 & ICHUM-1609 & D. 3 / 13 / 13 & sect. & - & & - \\
\hline 3.76 & ICHUM-1610 & D. $3 / 12 / 12$ & sect. & - & & - \\
\hline 3.77 & ICHUM-1611 & D. 3 / $11 / 11$ & sect. & - & & - \\
\hline 3.78 & ICHUM-1612 & D. $3 / 10 / 10$ & sect. & - & & - \\
\hline 3.79 & ICHUM-1613 & D. $3 / 9 / 9$ & sect. & - & & - \\
\hline 3. 80 & ICHUM-1614 & D. $3 / 8 / 8$ & sect. & - & & - \\
\hline 3.81 & ICHUM-1615 & D. $3 / 7 / 7$ & sect. & - & & - \\
\hline $3.82 *$ & ICHUM-1616 & $\mathrm{F} 6 * *$ & WM & $\begin{array}{l}\text { Cirrodrilus suzukii } \\
\text { (Yamaguchi, 1934) }\end{array}$ & syntype & $\begin{array}{l}\text { Suzuki- } \\
\text { zariganimimizu }\end{array}$ \\
\hline 3.83 & ICHUM-1617 & F $5 * *$ & WM & $\begin{array}{l}\text { Cirrodrilus uchidai } \\
\text { (Yamaguchi, 1932)? }\end{array}$ & syntype? & $\begin{array}{l}\text { Uchida- } \\
\text { zariganimimizu } \\
?\end{array}$ \\
\hline 3. 84 & ICHUM-1618 & $\mathrm{F} 4 * *$ & WM & $\begin{array}{l}\text { Cirrodrilus uchidai } \\
\text { (Yamaguchi, 1932)? }\end{array}$ & syntype ? & $\begin{array}{l}\text { Uchida- } \\
\text { zariganimimizu }\end{array}$ \\
\hline 3.85 & ICHUM-1619 & $\mathrm{F} 3 * *$ & WM & $\begin{array}{l}\text { Cirrodrilus uchidai } \\
\text { (Yamaguchi, 1932)? }\end{array}$ & syntype ? & $\begin{array}{l}\text { Uchida- } \\
\text { zariganimimizu }\end{array}$ \\
\hline $3.86 *$ & ICHUM-1620 & $\mathrm{F} 2 * *$ & WM & $\begin{array}{l}\text { Cirrodrilus uchidai } \\
\text { (Yamaguchi, 1932)? }\end{array}$ & syntype ? & $\begin{array}{l}\text { Uchida- } \\
\text { zariganimimizu }\end{array}$ \\
\hline 3. 87 & ICHUM-1621 & G. 4 & WM & $\begin{array}{l}\text { Cirrodrilus uchidai } \\
\text { (Yamaguchi, 1932)? }\end{array}$ & syntype ? & $\begin{array}{l}\text { Uchida- } \\
\text { zariganimimizu }\end{array}$ \\
\hline 3.88 & ICHUM-1622 & G. $5 * *$ & WM & $\begin{array}{l}\text { Cirrodrilus inukaii } \\
\text { (Yamaguchi, 1934) }\end{array}$ & syntype & $\begin{array}{l}\text { Inukai- } \\
\text { zariganimimizu }\end{array}$ \\
\hline 3.89 & ICHUM-1623 & B. $11 * *$ & WM & $\begin{array}{l}\text { Cirrodrilus uchidai } \\
\text { (Yamaguchi, 1932)? }\end{array}$ & syntype? & $\begin{array}{l}\text { Uchida- } \\
\text { zariganimimizu }\end{array}$ \\
\hline 3.90 & ICHUM-1624 & B. 6 & WM 1 & unidentified & & - \\
\hline$"$ & ICHUM-1625 & $"$ & WM 2 & $\begin{array}{l}\text { Cirrodrilus uchidai } \\
\text { (Yamaguchi, 1932)? }\end{array}$ & syntype? & $\begin{array}{l}\text { Uchida- } \\
\text { zariganimimizu }\end{array}$ \\
\hline 3.91 & ICHUM-1626 & B. 5 & WM 1 & $\begin{array}{l}\text { Cirrodrilus uchidai } \\
\text { (Yamaguchi, 1932)? }\end{array}$ & syntype? & $\begin{array}{l}\text { Uchida- } \\
\text { zariganimimizu }\end{array}$ \\
\hline " & ICHUM-1627 & $"$ & WM 2 & unidentified & & - \\
\hline$"$ & ICHUM-1628 & $"$ & WM 3 & unidentified & & - \\
\hline
\end{tabular}

...Continued on the next page 
TABLE 1. (Continued)

\begin{tabular}{|c|c|c|c|c|c|c|}
\hline $\begin{array}{l}\text { Box / } \\
\text { Slide } \\
\text { No. }\end{array}$ & Catalog No. & Slide Markings & $\begin{array}{l}\text { Specimen(s) } \\
\text { Status }\end{array}$ & Species Ident. & Type Status & $\begin{array}{l}\text { Japanese } \\
\text { common name }\end{array}$ \\
\hline 3.92 & ICHUM-1629 & B. $10 * *$ & WM & $\begin{array}{l}\text { Cirrodrilus uchidai } \\
\text { (Yamaguchi, 1932)? }\end{array}$ & syntype? & $\begin{array}{l}\text { Uchida- } \\
\text { zariganimimizu }\end{array}$ \\
\hline $3.93 *$ & ICHUM-1630 & $\mathrm{F} 7 * *$ & WM & $\begin{array}{l}\text { Cirrodrilus uchidai } \\
\text { (Yamaguchi, 1932)? }\end{array}$ & syntype? & $\begin{array}{l}\text { Uchida- } \\
\text { zariganimimizu }\end{array}$ \\
\hline $3.94 *$ & ICHUM-1631 & G. $1 * *$ & WM & $\begin{array}{l}\text { Cirrodrilus uchidai } \\
\text { (Yamaguchi, 1932)? }\end{array}$ & syntype? & $\begin{array}{l}\text { Uchida- } \\
\text { zariganimimizu }\end{array}$ \\
\hline 3.95 & ICHUM-1632 & G. $3 * *$ & WM & $\begin{array}{l}\text { Cirrodrilus uchidai } \\
\text { (Yamaguchi, 1932)? }\end{array}$ & syntype ? & $\begin{array}{l}\text { Uchida- } \\
\text { zariganimimizu }\end{array}$ \\
\hline 3.96 & ICHUM-1633 & D. 1 - 3 & sect. & - & & - \\
\hline $3.97 *$ & ICHUM-1634 & $\mathrm{A}^{\prime} 35 * *$ & WM & $\begin{array}{l}\text { Cirrodrilus uchidai } \\
\text { (Yamaguchi, 1932)? }\end{array}$ & syntype? & $\begin{array}{l}\text { Uchida- } \\
\text { zariganimimizu }\end{array}$ \\
\hline 3.98 & ICHUM-1635 & $\mathrm{A}^{\prime} 34 * *$ & WM & $\begin{array}{l}\text { Cirrodrilus uchidai } \\
\text { (Yamaguchi, 1932)? }\end{array}$ & syntype ? & $\begin{array}{l}\text { Uchida- } \\
\text { zariganimimizu } \\
?\end{array}$ \\
\hline 3.99 & ICHUM-1636 & A $33 * *$ & WM & $\begin{array}{l}\text { Cirrodrilus uchidai } \\
\text { (Yamaguchi, 1932)? }\end{array}$ & syntype? & $\begin{array}{l}\text { Uchida- } \\
\text { zariganimimizu }\end{array}$ \\
\hline 3. 100 & ICHUM-1637 & A $32 * *$ & WM & $\begin{array}{l}\text { Cirrodrilus uchidai } \\
\text { (Yamaguchi, 1932)? }\end{array}$ & syntype? & $\begin{array}{l}\text { Uchida- } \\
\text { zariganimimizu } \\
?\end{array}$ \\
\hline 4. $2 *$ & ICHUM-1638 & $215 / 2$ & WM 1 & $\begin{array}{l}\text { Branchiobdella } \\
\text { kobayashii } \\
\text { Yamaguchi, } 1934\end{array}$ & syntype? & $\begin{array}{l}\text { Kobayashi- } \\
\text { hirumimizu }\end{array}$ \\
\hline " & ICHUM-1639 & $"$ & WM 2 & $\begin{array}{l}\text { Branchiobdella } \\
\text { kobayashii } \\
\text { Yamaguchi, } 1934\end{array}$ & syntype? & $\begin{array}{l}\text { Kobayashi- } \\
\text { hirumimizu }\end{array}$ \\
\hline 4.3 & ICHUM-1640 & $215 / 3$ & WM & unidentified & & - \\
\hline 4.4 & ICHUM-1641 & $215 / 4$ & WM & $\begin{array}{l}\text { Cirrodrilus inukaii } \\
\text { (Yamaguchi, 1934)? }\end{array}$ & syntype & $\begin{array}{l}\text { Inukai- } \\
\text { zariganimimizu } \\
?\end{array}$ \\
\hline 4. $5 *$ & ICHUM-1642 & $215 / 5$ & WM & $\begin{array}{l}\text { Cirrodrilus } \\
\text { kawamurai } \\
\text { (Yamaguchi, 1934) }\end{array}$ & syntype? & $\begin{array}{l}\text { Kawamura- } \\
\text { zariganimimizu }\end{array}$ \\
\hline 4. 6 & ICHUM-1643 & $215 / 6$ & WM & unidentified & & - \\
\hline 4.7 & ICHUM-1644 & $215 / 7$ & WM & unidentified & & - \\
\hline 4. $8 *$ & ICHUM-1645 & $215 / 8$ & WM & $\begin{array}{l}\text { Branchiobdella } \\
\text { monodontus Liu \& } \\
\text { Zhang, } 1983\end{array}$ & non-type & - \\
\hline
\end{tabular}

...Continued on the next page 
TABLE 1. (Continued)

\begin{tabular}{|c|c|c|c|c|c|c|}
\hline $\begin{array}{l}\text { Box / } \\
\text { Slide } \\
\text { No. }\end{array}$ & Catalog No. & Slide Markings & $\begin{array}{l}\text { Specimen(s) } \\
\text { Status }\end{array}$ & Species Ident. & Type Status & $\begin{array}{l}\text { Japanese } \\
\text { common name }\end{array}$ \\
\hline 4. $9 *$ & ICHUM-1646 & $215 / 9$ & WM & $\begin{array}{l}\text { Branchiobdella } \\
\text { monodontus Liu \& } \\
\text { Zhang, } 1983\end{array}$ & non-type & - \\
\hline $4.12 *$ & ICHUM-1647 & $215 / 12$ & WM & $\begin{array}{l}\text { Branchiobdella } \\
\text { kobayashii } \\
\text { Yamaguchi, } 1934\end{array}$ & syntype? & $\begin{array}{l}\text { Kobayashi- } \\
\text { hirumimizu }\end{array}$ \\
\hline 4. 13 & ICHUM-1648 & $215 / 13$ & WM & unidentified & & - \\
\hline $4.14 *$ & ICHUM-1649 & $215 / 14$ & WM & $\begin{array}{l}\text { Holtodrilus truncatus } \\
\text { (Liang, 1963) }\end{array}$ & non-type & $\begin{array}{l}\text { Ebi- } \\
\text { yadorimimizu }\end{array}$ \\
\hline $4.15 *$ & ICHUM-1650 & $215 / 15$ & WM & $\begin{array}{l}\text { Holtodrilus truncatus } \\
\text { (Liang, 1963) }\end{array}$ & non-type & $\begin{array}{l}\text { Ebi- } \\
\text { yadorimimizu }\end{array}$ \\
\hline $4.16 *$ & ICHUM-1651 & $215 / 16$ & WM & $\begin{array}{l}\text { Branchiobdella } \\
\text { kobayashii } \\
\text { Yamaguchi, } 1934\end{array}$ & syntype? & $\begin{array}{l}\text { Kobayashi- } \\
\text { hirumimizu }\end{array}$ \\
\hline 4. 18 & ICHUM-1652 & $215 / 18$ & WM & $\begin{array}{l}\text { Holtodrilus truncatus } \\
\text { (Liang, 1963) }\end{array}$ & non-type & $\begin{array}{l}\text { Ebi- } \\
\text { yadorimimizu }\end{array}$ \\
\hline 4. 19 & ICHUM-1653 & 230 & WM & oligochaete & & - \\
\hline 4. $21 *$ & ICHUM-1654 & 21 & WM 1-7 & unidentified & & - \\
\hline 4. 31 & ICHUM-1655 & c.s. 25 & WM 1 & $\begin{array}{l}\text { Cirrodrilus } \\
\text { aomorensis } \\
\text { (Yamaguchi, 1934) }\end{array}$ & syntype & $\begin{array}{l}\text { Aomori- } \\
\text { zariganimimizu }\end{array}$ \\
\hline " & ICHUM-1656 & $"$ & WM 2 & $\begin{array}{l}\text { Cirrodrilus } \\
\text { aomorensis } \\
\text { (Yamaguchi, 1934) }\end{array}$ & syntype & $\begin{array}{l}\text { Aomori- } \\
\text { zariganimimizu }\end{array}$ \\
\hline$"$ & ICHUM-1657 & $"$ & WM 3 & unidentified & & - \\
\hline 4. 34 & ICHUM-1658 & 34 & WM & unidentified & & - \\
\hline 4. 36 & ICHUM-1659 & $\begin{array}{l}\text { c s. }-25 / \text { sp. c.s. /D } \\
3\end{array}$ & WM & $\begin{array}{l}\text { Cirrodrilus inukaii } \\
\text { (Yamaguchi, 1934)? }\end{array}$ & syntype & $\begin{array}{l}\text { Inukai- } \\
\text { zariganimimizu } \\
?\end{array}$ \\
\hline 4. 38 & ICHUM-1660 & $\begin{array}{l}\text { c.s - 23/ sp. c.s /D. } \\
5\end{array}$ & WM 1 & $\begin{array}{l}\text { Cirrodrilus inukaii } \\
\text { (Yamaguchi, 1934)? }\end{array}$ & syntype & $\begin{array}{l}\text { Inukai- } \\
\text { zariganimimizu } \\
?\end{array}$ \\
\hline$"$ & ICHUM-1661 & $"$ & WM 2 & $\begin{array}{l}\text { Cirrodrilus inukaii } \\
\text { (Yamaguchi, 1934)? }\end{array}$ & syntype & $\begin{array}{l}\text { Inukai- } \\
\text { zariganimimizu } \\
?\end{array}$ \\
\hline " & ICHUM-1662 & $"$ & WM 3 & $\begin{array}{l}\text { Cirrodrilus inukaii } \\
\text { (Yamaguchi, 1934) }\end{array}$ & syntype & $\begin{array}{l}\text { Inukai- } \\
\text { zariganimimizu }\end{array}$ \\
\hline
\end{tabular}

...Continued on the next page 
TABLE 1. (Continued)

\begin{tabular}{|c|c|c|c|c|c|c|}
\hline $\begin{array}{l}\text { Box / } \\
\text { Slide } \\
\text { No. }\end{array}$ & Catalog No. & Slide Markings & $\begin{array}{l}\text { Specimen(s) } \\
\text { Status }\end{array}$ & Species Ident. & Type Status & $\begin{array}{l}\text { Japanese } \\
\text { common name }\end{array}$ \\
\hline 4. 40 & ICHUM-1663 & c. -27 & WM & $\begin{array}{l}\text { Cirrodrilus inukaii } \\
\text { (Yamaguchi, 1934) }\end{array}$ & syntype & $\begin{array}{l}\text { Inukai- } \\
\text { zariganimimizu }\end{array}$ \\
\hline 4. 42 & ICHUM-1664 & $\begin{array}{l}\text { c s. }-20 / \text { sp. c.s /D } \\
1\end{array}$ & WM 1-3 & unidentified & & - \\
\hline 4. 43 & ICHUM-1665 & $\begin{array}{l}\text { c.s - 21/ Sp. c.s /D } \\
8\end{array}$ & WM 1 & $\begin{array}{l}\text { Cirrodrilus inukaii } \\
\text { (Yamaguchi, 1934)? }\end{array}$ & syntype & $\begin{array}{l}\text { Inukai- } \\
\text { zariganimimizu } \\
?\end{array}$ \\
\hline$"$ & ICHUM-1666 & $"$ & WM 2 & $\begin{array}{l}\text { Cirrodrilus ezoensis } \\
\text { (Yamaguchi, 1934) }\end{array}$ & syntype & $\begin{array}{l}\text { Ezo- } \\
\text { zariganimimizu }\end{array}$ \\
\hline$"$ & ICHUM-1667 & $"$ & WM 3 & $\begin{array}{l}\text { Cirrodrilus inukaii } \\
\text { (Yamaguchi, 1934)? }\end{array}$ & syntype & $\begin{array}{l}\text { Inukai- } \\
\text { zariganimimizu } \\
?\end{array}$ \\
\hline 4. 45 & ICHUM-1668 & 45 & sect. & - & & - \\
\hline 4. 46 & ICHUM-1669 & 8 & sect. & - & & - \\
\hline 4. 48 & ICHUM-1670 & K. 32 & WM & destroyed & & - \\
\hline 4. 49 & ICHUM-1671 & 12 & WM & unidentified & & - \\
\hline 4. 69 & ICHUM-1672 & 69 & WM & unidentified & & - \\
\hline 4. 71 & ICHUM-1673 & 71 & WM & unidentified & & - \\
\hline 4. 73 & ICHUM-1674 & 73 & sect. & - & & - \\
\hline 4. 74 & ICHUM-1675 & 10 & sect. & - & & - \\
\hline 4. 76 & ICHUM-1676 & 76 & sect. & destroyed & & - \\
\hline 4. 77 & ICHUM-1677 & 14 & sect. & - & & - \\
\hline 4. 78 & ICHUM-1678 & 78 & sect. & - & & - \\
\hline 4. 82 & ICHUM-1679 & 11 & sect. & - & & - \\
\hline 4. 86 & ICHUM-1680 & 86 & sect. & - & & - \\
\hline 4.92 & ICHUM-1681 & 13 & sect. & - & & - \\
\hline 4. 97 & ICHUM-1682 & 97 & sect. & - & & - \\
\hline 5.1 & ICHUM-1683 & $\begin{array}{l}2 \text { / Branch. } \\
\text { Pentadonta }\end{array}$ & sect. & - & & - \\
\hline 5.2 & ICHUM-1684 & $\begin{array}{l}3 \text { / Branch. } \\
\text { Pentadonta }\end{array}$ & sect. & - & & - \\
\hline 5.3 & ICHUM-1685 & $\begin{array}{l}3 \text { A / Branch. } \\
\text { Pentadonta }\end{array}$ & sect. & - & & - \\
\hline
\end{tabular}


TABLE 1. (Continued)

\begin{tabular}{|c|c|c|c|c|c|c|}
\hline $\begin{array}{l}\text { Box / } \\
\text { Slide } \\
\text { No. }\end{array}$ & Catalog No. & Slide Markings & $\begin{array}{l}\text { Specimen(s) } \\
\text { Status }\end{array}$ & Species Ident. & Type Status & $\begin{array}{l}\text { Japanese } \\
\text { common name }\end{array}$ \\
\hline 5.4 & ICHUM-1686 & K. $1 / 6$ & WM & $\begin{array}{l}\text { Branchiobdella } \\
\text { macroperistomium } \\
\text { Liu \& Zhang, } 1983 ?\end{array}$ & non-type & - \\
\hline 5.8 & ICHUM-1687 & K. $1.6 / \mathrm{K} 1$ & sect. & - & & - \\
\hline 5.11 & ICHUM-1688 & K. 1.8 & sect. & - & & - \\
\hline 5.13 & ICHUM-1689 & K. 1.9 & sect. & - & & - \\
\hline 5. 14 & ICHUM-1690 & K. 1.10 & sect. & - & & - \\
\hline $5.15 *$ & ICHUM-1691 & K. $1 / 11$ & $\mathrm{WM}$ & $\begin{array}{l}\text { Branchiobdella } \\
\text { kobayashii } \\
\text { Yamaguchi, } 1934\end{array}$ & syntype? & $\begin{array}{l}\text { Kobayashi- } \\
\text { hirumimizu }\end{array}$ \\
\hline $5.16 *$ & ICHUM-1692 & K. 1.12 & $\mathrm{WM}$ & $\begin{array}{l}\text { Branchiobdella } \\
\text { kobayashii } \\
\text { Yamaguchi, } 1934\end{array}$ & syntype? & $\begin{array}{l}\text { Kobayashi- } \\
\text { hirumimizu }\end{array}$ \\
\hline $5.18 *$ & ICHUM-1693 & K. 1.14 / K. $1 / \mathrm{D}$ & WM & $\begin{array}{l}\text { Branchiobdella } \\
\text { macroperistomium } \\
\text { Liu \& Zhang, } 1983 ?\end{array}$ & non-type & - \\
\hline 5. 19 & ICHUM-1694 & K $1 . / 15 / \mathrm{K} 1 . / \mathrm{D}$ & WM & $\begin{array}{l}\text { Branchiobdella } \\
\text { kobayashii } \\
\text { Yamaguchi, } 1934\end{array}$ & syntype? & $\begin{array}{l}\text { Kobayashi- } \\
\text { hirumimizu }\end{array}$ \\
\hline 5. 20 & ICHUM-1695 & K. $1 . / 13$ & $\mathrm{WM}$ & $\begin{array}{l}\text { Branchiobdella } \\
\text { kobayashii } \\
\text { Yamaguchi, 1934? }\end{array}$ & syntype? & $\begin{array}{l}\text { Kobayashi- } \\
\text { hirumimizu? }\end{array}$ \\
\hline 5.21 & ICHUM-1696 & K 1.4 / K 1 & sect. & - & & - \\
\hline 5.24 & ICHUM-1697 & K. $1 / 19$ & sect. & - & & - \\
\hline 5. 28 & ICHUM-1698 & K. 2 - 4 & sect. & - & & - \\
\hline 5.31 & ICHUM-1699 & K $2 / 2 / K .2-5-2$ & sect. & - & & - \\
\hline 5.33 & ICHUM-1700 & K $2 / 1 / K .2-5-1$ & sect. & - & & - \\
\hline 5.34 & ICHUM-1701 & K $2 / 17$ & sect. & - & & - \\
\hline 5.37 & ICHUM-1702 & K. 2 & sect. & - & & - \\
\hline 5. 40 & ICHUM-1703 & K $3 / 17$ - 2 & sect. & - & & - \\
\hline 5.42 & ICHUM-1704 & K $3 / 17-1$ & sect. & - & & - \\
\hline 5.44 & ICHUM-1705 & K. 3 / 8 & WM & $\begin{array}{l}\text { Cirrodrilus } \\
\text { kawamurai } \\
\text { (Yamaguchi, 1934) }\end{array}$ & syntype? & $\begin{array}{l}\text { Kawamura- } \\
\text { zariganimimizu }\end{array}$ \\
\hline
\end{tabular}

...Continued on the next page 
TABLE 1. (Continued)

\begin{tabular}{|c|c|c|c|c|c|c|}
\hline $\begin{array}{l}\text { Box / } \\
\text { Slide } \\
\text { No. }\end{array}$ & Catalog No. & Slide Markings & $\begin{array}{l}\text { Specimen(s) } \\
\text { Status }\end{array}$ & Species Ident. & Type Status & $\begin{array}{l}\text { Japanese } \\
\text { common name }\end{array}$ \\
\hline 5.45 & ICHUM-1706 & K. $3 / 10 /$ K. $3 / \mathrm{D}$ & WM & $\begin{array}{l}\text { Cirrodrilus } \\
\text { kawamurai } \\
\text { (Yamaguchi, 1934) }\end{array}$ & syntype ? & $\begin{array}{l}\text { Kawamura- } \\
\text { zariganimimizu }\end{array}$ \\
\hline 5.46 & ICHUM-1707 & K $3 / 9$ / K $3 / D$ & WM & $\begin{array}{l}\text { Cirrodrilus } \\
\text { kawamurai } \\
\text { (Yamaguchi, 1934) }\end{array}$ & syntype ? & $\begin{array}{l}\text { Kawamura- } \\
\text { zariganimimizu }\end{array}$ \\
\hline $5.47 *$ & ICHUM-1708 & K. $3 / 11 / \mathrm{K} 3 / \mathrm{D}$ & WM & $\begin{array}{l}\text { Cirrodrilus } \\
\text { kawamurai } \\
\text { (Yamaguchi, 1934) }\end{array}$ & syntype? & $\begin{array}{l}\text { Kawamura- } \\
\text { zariganimimizu }\end{array}$ \\
\hline $5.48 *$ & ICHUM-1709 & K $3 / 2$ & WM & $\begin{array}{l}\text { Cirrodrilus } \\
\text { kawamurai } \\
\text { (Yamaguchi, 1934) }\end{array}$ & syntype? & $\begin{array}{l}\text { Kawamura- } \\
\text { zariganimimizu }\end{array}$ \\
\hline 5.49 & ICHUM-1710 & K. 3 / 12 & $\mathrm{WM}$ & $\begin{array}{l}\text { Cirrodrilus } \\
\text { kawamurai } \\
\text { (Yamaguchi, 1934) }\end{array}$ & syntype? & $\begin{array}{l}\text { Kawamura- } \\
\text { zariganimimizu }\end{array}$ \\
\hline 5.50 & ICHUM-1711 & K. 3 / 13 / K 3 / D & WM & $\begin{array}{l}\text { Cirrodrilus } \\
\text { kawamurai } \\
\text { (Yamaguchi, 1934) }\end{array}$ & syntype ? & $\begin{array}{l}\text { Kawamura- } \\
\text { zariganimimizu }\end{array}$ \\
\hline $5.51 *$ & ICHUM-1712 & K. 3 / 14 & WM & $\begin{array}{l}\text { Cirrodrilus } \\
\text { kawamurai } \\
\text { (Yamaguchi, 1934) }\end{array}$ & syntype? & $\begin{array}{l}\text { Kawamura- } \\
\text { zariganimimizu }\end{array}$ \\
\hline 5.52 & ICHUM-1713 & K. $3 / 15$ & WM & $\begin{array}{l}\text { Cirrodrilus } \\
\text { kawamurai } \\
\text { (Yamaguchi, 1934) }\end{array}$ & syntype? & $\begin{array}{l}\text { Kawamura- } \\
\text { zariganimimizu }\end{array}$ \\
\hline 5.58 & ICHUM-1714 & K. 6 & sect. & - & & - \\
\hline 5. 59 & ICHUM-1715 & K. 5 - 4 - 2 & sect. & - & & - \\
\hline 5.60 & ICHUM-1716 & $\begin{array}{l}\text { K. } 5 \text { - } 4 \text { - 3/ K } 5 / 1- \\
3\end{array}$ & sect. & - & & - \\
\hline 5.61 & ICHUM-1717 & K. $4-10-3$ & sect. & - & & - \\
\hline 5.62 & ICHUM-1718 & K. 4 - 6 & sect. & - & & - \\
\hline 5.63 & ICHUM-1719 & K. $3-6-4$ / K 3 & sect. & - & & - \\
\hline 5.64 & ICHUM-1720 & K. $3 / 5$ & sect. & - & & - \\
\hline 5.65 & ICHUM-1721 & K. 4 - 5 & sect. & - & & - \\
\hline 5.66 & ICHUM-1722 & K 4 / 4 & WM & unidentified & & - \\
\hline 5.67 & ICHUM-1723 & $\mathrm{K} 4 / 8$ & WM & unidentified & & - \\
\hline
\end{tabular}


TABLE 1. (Continued)

\begin{tabular}{|c|c|c|c|c|c|c|}
\hline $\begin{array}{l}\text { Box / } \\
\text { Slide } \\
\text { No. }\end{array}$ & Catalog No. & Slide Markings & $\begin{array}{l}\text { Specimen(s) } \\
\text { Status }\end{array}$ & Species Ident. & Type Status & $\begin{array}{l}\text { Japanese } \\
\text { common name }\end{array}$ \\
\hline 5.68 & ICHUM-1724 & K. 4 / 9 & WM & $\begin{array}{l}\text { Branchiobdella } \\
\text { orientalis } \\
\text { Yamaguchi, } 1934\end{array}$ & syntype? & Oo-hirumimizu \\
\hline 5.69 & ICHUM-1725 & K. 4 / 10 & $\mathrm{WM}$ & $\begin{array}{l}\text { Branchiobdella } \\
\text { orientalis } \\
\text { Yamaguchi, } 1934\end{array}$ & syntype? & Oo-hirumimizu \\
\hline 5.70 & ICHUM-1726 & K $3 / 16-1$ & sect. & - & & - \\
\hline 5.72 & ICHUM-1727 & K. 2. - 3 & sect. & - & & - \\
\hline 5.73 & ICHUM-1728 & $\begin{array}{l}\text { 1./ Branchio. } \\
\text { Pentadonta }\end{array}$ & sect. & - & & - \\
\hline 5.75 & ICHUM-1729 & K. $4.4-2$ & sect. & - & & - \\
\hline 5.76 & ICHUM-1730 & K $3 / \mathrm{K} 3-6-3$ & sect. & - & & - \\
\hline 5.77 & ICHUM-1731 & K $3.16-2$ & sect. & - & & - \\
\hline 5.78 & ICHUM-1732 & K. $4.4-1 / 1-1$ & sect. & - & & - \\
\hline 5.79 & ICHUM-1733 & K. $4 / 2 .-3$ & sect. & - & & - \\
\hline 5.80 & ICHUM-1734 & K. $4 / 2.2-3$ & sect. & - & & - \\
\hline 5.81 & ICHUM-1735 & K 5 / K. 5 / 5 & sect. & - & & - \\
\hline 5.82 & ICHUM-1736 & K 3 / K. 3 - 6 - 1 & sect. & - & & - \\
\hline 5.83 & ICHUM-1737 & K 3 / K 3. - $6-2$ & sect. & - & & - \\
\hline 5.85 & ICHUM-1738 & K. $4-11-1$ & sect. & - & & - \\
\hline 5.86 & ICHUM-1739 & K. 4 -11- 2 & sect. & - & & - \\
\hline 5.87 & ICHUM-1740 & K. 5.13 - 2 & sect. & - & & - \\
\hline 5. 88 & ICHUM-1741 & K $5 / 13-4$ & sect. & - & & - \\
\hline 5.89 & ICHUM-1742 & K. $5-13-1$ & sect. & - & & - \\
\hline 5.90 & ICHUM-1743 & $\mathrm{K} 4 / 2.1-2$ & sect. & - & & - \\
\hline 5.91 & ICHUM-1744 & $\mathrm{K} 4 / 3.1-1 / 2.1$ & sect. & - & & - \\
\hline 5.93 & ICHUM-1745 & K 5. - $4-1$ & sect. & - & & - \\
\hline 5.94 & ICHUM-1746 & K. 4. - $11-3$ & sect. & - & & - \\
\hline 5.95 & ICHUM-1747 & K. $5 / 5$ & sect. & - & & - \\
\hline $5.96 *$ & ICHUM-1748 & K $2 / 6$ & WM 1 & $\begin{array}{l}\text { Cirrodrilus chosen } \\
\text { (Yamaguchi, 1934) }\end{array}$ & syntype & $\begin{array}{l}\text { Chosen- } \\
\text { zariganimimizu }\end{array}$ \\
\hline
\end{tabular}

...Continued on the next page 
TABLE 1. (Continued)

\begin{tabular}{|c|c|c|c|c|c|c|}
\hline $\begin{array}{l}\text { Box / } \\
\text { Slide } \\
\text { No. }\end{array}$ & Catalog No. & Slide Markings & $\begin{array}{l}\text { Specimen(s) } \\
\text { Status }\end{array}$ & Species Ident. & Type Status & $\begin{array}{l}\text { Japanese } \\
\text { common name }\end{array}$ \\
\hline$"$ & ICHUM-1749 & $"$ & WM 2 & $\begin{array}{l}\text { Cirrodrilus chosen } \\
\text { (Yamaguchi, 1934) }\end{array}$ & syntype & $\begin{array}{l}\text { Chosen- } \\
\text { zariganimimizu }\end{array}$ \\
\hline$"$ & ICHUM-1750 & $"$ & WM 3 & $\begin{array}{l}\text { Cirrodrilus chosen } \\
\text { (Yamaguchi, 1934) }\end{array}$ & syntype & $\begin{array}{l}\text { Chosen- } \\
\text { zariganimimizu }\end{array}$ \\
\hline 5.97 & ICHUM-1751 & K $5 / 7$ & WM & $\begin{array}{l}\text { Cirrodrilus suzukii } \\
\text { (Yamaguchi, 1934) }\end{array}$ & syntype? & $\begin{array}{l}\text { Suzuki- } \\
\text { zariganimimizu }\end{array}$ \\
\hline $5.98 *$ & ICHUM-1752 & K. $5 / 8$ & WM & $\begin{array}{l}\text { Cirrodrilus chosen } \\
\text { (Yamaguchi, 1934) }\end{array}$ & syntype & $\begin{array}{l}\text { Chosen- } \\
\text { zariganimimizu }\end{array}$ \\
\hline $5.99 *$ & ICHUM-1753 & K 5 / 9 & WM & $\begin{array}{l}\text { Cirrodrilus suzukii } \\
\text { (Yamaguchi, 1934) }\end{array}$ & syntype ? & $\begin{array}{l}\text { Suzuki- } \\
\text { zariganimimizu }\end{array}$ \\
\hline 5.100 & ICHUM-1754 & K. 5 / 11 & WM & $\begin{array}{l}\text { Cirrodrilus suzukii } \\
\text { (Yamaguchi, 1934) }\end{array}$ & syntype ? & $\begin{array}{l}\text { Suzuki- } \\
\text { zariganimimizu }\end{array}$ \\
\hline 6.1 & ICHUM-1755 & am A / 2 & sect. & - & & - \\
\hline 6.2 & ICHUM-1756 & am B / 4 & sect. & - & & - \\
\hline 6.3 & ICHUM-1757 & am A / 1 & sect. & - & & - \\
\hline 6.4 & ICHUM-1758 & am A / 5 & sect. & - & & - \\
\hline 6.5 & ICHUM-1759 & am A / 6 & sect. & - & & - \\
\hline 6.6 & ICHUM-1760 & am A / 4 & sect. & - & & - \\
\hline 6.7 & ICHUM-1761 & am. B / 5 & sect. & - & & - \\
\hline 6.8 & ICHUM-1762 & am A / 3 & sect. & - & & - \\
\hline 6.9 & ICHUM-1763 & am B / 1 & sect. & - & & - \\
\hline 6. 10 & ICHUM-1764 & am. B / 2 & sect. & - & & - \\
\hline 6.11 & ICHUM-1765 & am B / 3 & sect. & - & & - \\
\hline 6.12 & ICHUM-1766 & am A / 7 & sect. & - & & - \\
\hline 6.13 & ICHUM-1767 & am B / 6 & sect. & - & & - \\
\hline 6.21 & ICHUM-1768 & $\mathrm{AO} / 1$ & WM & $\begin{array}{l}\text { Cirrodrilus } \\
\text { aomorensis } \\
\text { (Yamaguchi, 1934) }\end{array}$ & syntype & $\begin{array}{l}\text { Aomori- } \\
\text { zariganimimizu }\end{array}$ \\
\hline 6.24 & ICHUM-1769 & $\mathrm{AO} / 2$ & WM & $\begin{array}{l}\text { Cirrodrilus } \\
\text { aomorensis } \\
\text { (Yamaguchi, 1934) }\end{array}$ & syntype & $\begin{array}{l}\text { Aomori- } \\
\text { zariganimimizu }\end{array}$ \\
\hline 6.25 & ICHUM-1770 & $\mathrm{AO} / 3$ & WM & $\begin{array}{l}\text { Cirrodrilus } \\
\text { aomorensis } \\
\text { (Yamaguchi, 1934) }\end{array}$ & syntype & $\begin{array}{l}\text { Aomori- } \\
\text { zariganimimizu }\end{array}$ \\
\hline
\end{tabular}

...Continued on the next page 
TABLE 1. (Continued)

\begin{tabular}{|c|c|c|c|c|c|c|}
\hline $\begin{array}{l}\text { Box / } \\
\text { Slide } \\
\text { No. }\end{array}$ & Catalog No. & Slide Markings & $\begin{array}{l}\text { Specimen(s) } \\
\text { Status }\end{array}$ & Species Ident. & Type Status & $\begin{array}{l}\text { Japanese } \\
\text { common name }\end{array}$ \\
\hline $6.26 *$ & ICHUM-1771 & $\mathrm{AO} / 4$ & WM 1 & $\begin{array}{l}\text { Cirrodrilus } \\
\text { aomorensis } \\
\text { (Yamaguchi, 1934) }\end{array}$ & syntype & $\begin{array}{l}\text { Aomori- } \\
\text { zariganimimizu }\end{array}$ \\
\hline " & ICHUM-1772 & $"$ & WM 2 & $\begin{array}{l}\text { Cirrodrilus } \\
\text { aomorensis } \\
\text { (Yamaguchi, 1934) }\end{array}$ & syntype & $\begin{array}{l}\text { Aomori- } \\
\text { zariganimimizu }\end{array}$ \\
\hline $6.27 *$ & ICHUM-1773 & 27 & WM 1 & $\begin{array}{l}\text { Cirrodrilus inukaii } \\
\text { (Yamaguchi, 1934) }\end{array}$ & syntype & $\begin{array}{l}\text { Inukai- } \\
\text { zariganimimizu }\end{array}$ \\
\hline " & ICHUM-1774 & $"$ & WM 2 & $\begin{array}{l}\text { Cirrodrilus inukaii } \\
\text { (Yamaguchi, 1934) }\end{array}$ & syntype & $\begin{array}{l}\text { Inukai- } \\
\text { zariganimimizu }\end{array}$ \\
\hline " & ICHUM-1775 & $"$ & WM 3 & $\begin{array}{l}\text { Cirrodrilus } \\
\text { aomorensis } \\
\text { (Yamaguchi, 1934) }\end{array}$ & syntype & $\begin{array}{l}\text { Aomori- } \\
\text { zariganimimizu }\end{array}$ \\
\hline " & ICHUM-1776 & $"$ & WM 4 & $\begin{array}{l}\text { Cirrodrilus } \\
\text { aomorensis } \\
\text { (Yamaguchi, 1934) }\end{array}$ & syntype & $\begin{array}{l}\text { Aomori- } \\
\text { zariganimimizu }\end{array}$ \\
\hline$"$ & ICHUM-1777 & $"$ & WM 5 & $\begin{array}{l}\text { Cirrodrilus } \\
\text { aomorensis } \\
\text { (Yamaguchi, 1934) }\end{array}$ & syntype & $\begin{array}{l}\text { Aomori- } \\
\text { zariganimimizu }\end{array}$ \\
\hline " & ICHUM-1778 & " & WM 6 & $\begin{array}{l}\text { Cirrodrilus } \\
\text { aomorensis } \\
\text { (Yamaguchi, 1934) }\end{array}$ & syntype & $\begin{array}{l}\text { Aomori- } \\
\text { zariganimimizu }\end{array}$ \\
\hline 6.28 & ICHUM-1779 & $\mathrm{AO} / \mathrm{A} 2$ & WM & $\begin{array}{l}\text { Cirrodrilus inukaii } \\
\text { (Yamaguchi, 1934) }\end{array}$ & syntype & $\begin{array}{l}\text { Inukai- } \\
\text { zariganimimizu }\end{array}$ \\
\hline 6.29 & ICHUM-1780 & $\mathrm{AO} / 5$ & WM & $\begin{array}{l}\text { Cirrodrilus } \\
\text { aomorensis } \\
\text { (Yamaguchi, 1934) }\end{array}$ & syntype & $\begin{array}{l}\text { Aomori- } \\
\text { zariganimimizu }\end{array}$ \\
\hline 6.31 & ICHUM-1781 & $C-8$ & WM 1 & $\begin{array}{l}\text { Cirrodrilus suzukii } \\
\text { (Yamaguchi, 1934) }\end{array}$ & syntype ? & $\begin{array}{l}\text { Suzuki- } \\
\text { zariganimimizu }\end{array}$ \\
\hline$"$ & ICHUM-1782 & " & WM 2 & unidentified & & - \\
\hline$"$ & ICHUM-1783 & $"$ & WM 3 & unidentified & & - \\
\hline " & ICHUM-1784 & $"$ & WM 4 & $\begin{array}{l}\text { Cirrodrilus } \\
\text { megalodentatus } \\
\text { (Yamaguchi, 1934) }\end{array}$ & syntype & $\begin{array}{l}\text { Ooago- } \\
\text { zariganimimizu }\end{array}$ \\
\hline 6.32 & ICHUM-1785 & $\mathrm{K} 1 . / 5 / 51 / \mathrm{K} 1$ & sect. & - & & - \\
\hline 6.33 & ICHUM-1786 & c.d.s. 20 / c.d.s. & sect. & - & & - \\
\hline 6.34 & ICHUM-1787 & c.d.s / c.d s / 18 & sect. & - & & - \\
\hline 6.35 & ICHUM-1788 & c d s. 19 / c.d.s 5 & sect. & - & & - \\
\hline
\end{tabular}


TABLE 1. (Continued)

\begin{tabular}{|c|c|c|c|c|c|c|}
\hline $\begin{array}{l}\text { Box / } \\
\text { Slide } \\
\text { No. }\end{array}$ & Catalog No. & Slide Markings & $\begin{array}{l}\text { Specimen(s) } \\
\text { Status }\end{array}$ & Species Ident. & Type Status & $\begin{array}{l}\text { Japanese } \\
\text { common name }\end{array}$ \\
\hline $6.37 *$ & ICHUM-1789 & $\begin{array}{l}\text { Sp.c d 1/ D } 1 / \text { c.d s } \\
17\end{array}$ & WM & $\begin{array}{l}\text { Cirrodrilus } \\
\text { megalodentatus } \\
\text { (Yamaguchi, 1934) }\end{array}$ & syntype & $\begin{array}{l}\text { Ooago- } \\
\text { zariganimimizu }\end{array}$ \\
\hline 6.38 & ICHUM-1790 & c.d.s. 21 & sect. & - & & - \\
\hline 6.39 & ICHUM-1791 & 39 & WM & unidentified & & - \\
\hline 6.40 & ICHUM-1792 & 40 & WM & unidentified & & - \\
\hline 6.41 & ICHUM-1793 & 41 & WM 1-2 & unidentified & & - \\
\hline 6.42 & ICHUM-1794 & $\begin{array}{l}\text { c.s - 22/ Sp. c.s. /D } \\
2\end{array}$ & WM 1 & $\begin{array}{l}\text { Cirrodrilus inukaii } \\
\text { (Yamaguchi, 1934) }\end{array}$ & syntype & $\begin{array}{l}\text { Inukai- } \\
\text { zariganimimizu }\end{array}$ \\
\hline " & ICHUM-1795 & $"$ & WM 2 & $\begin{array}{l}\text { Cirrodrilus inukaii } \\
\text { (Yamaguchi, 1934) }\end{array}$ & syntype & $\begin{array}{l}\text { Inukai- } \\
\text { zariganimimizu }\end{array}$ \\
\hline$"$ & ICHUM-1796 & $"$ & WM 3 & $\begin{array}{l}\text { Cirrodrilus inukaii } \\
\text { (Yamaguchi, 1934) }\end{array}$ & syntype & $\begin{array}{l}\text { Inukai- } \\
\text { zariganimimizu }\end{array}$ \\
\hline 6.43 & ICHUM-1797 & c. -7 & WM 1 & unidentified & & - \\
\hline$"$ & ICHUM-1798 & $"$ & WM 2 & $\begin{array}{l}\text { Cirrodrilus } \\
\text { megalodentatus } \\
\text { (Yamaguchi, 1934) }\end{array}$ & syntype & $\begin{array}{l}\text { Ooago- } \\
\text { zariganimimizu }\end{array}$ \\
\hline$"$ & ICHUM-1799 & $"$ & WM 3 & $\begin{array}{l}\text { Cirrodrilus ezoensis } \\
\text { (Yamaguchi, 1934) }\end{array}$ & syntype & $\begin{array}{l}\text { Ezo- } \\
\text { zariganimimizu }\end{array}$ \\
\hline$"$ & ICHUM-1800 & $"$ & WM 4 & $\begin{array}{l}\text { Cirrodrilus } \\
\text { megalodentatus } \\
\text { (Yamaguchi, 1934) }\end{array}$ & syntype & $\begin{array}{l}\text { Ooago- } \\
\text { zariganimimizu }\end{array}$ \\
\hline$"$ & ICHUM-1801 & $"$ & WM 5 & $\begin{array}{l}\text { Cirrodrilus ezoensis } \\
\text { (Yamaguchi, 1934) }\end{array}$ & syntype & $\begin{array}{l}\text { Ezo- } \\
\text { zariganimimizu }\end{array}$ \\
\hline " & ICHUM-1802 & $"$ & WM 6 & $\begin{array}{l}\text { Cirrodrilus ezoensis } \\
\text { (Yamaguchi, 1934) }\end{array}$ & syntype & $\begin{array}{l}\text { Ezo- } \\
\text { zariganimimizu }\end{array}$ \\
\hline$"$ & ICHUM-1803 & $"$ & WM 7 & unidentified & & - \\
\hline$"$ & ICHUM-1804 & $"$ & WM 8 & $\begin{array}{l}\text { Cirrodrilus } \\
\text { megalodentatus } \\
\text { (Yamaguchi, 1934) }\end{array}$ & syntype & $\begin{array}{l}\text { Ooago- } \\
\text { zariganimimizu }\end{array}$ \\
\hline$"$ & ICHUM-1805 & $"$ & WM 9 & unidentified & & - \\
\hline$"$ & ICHUM-1806 & $"$ & WM 10 & $\begin{array}{l}\text { Cirrodrilus ezoensis } \\
\text { (Yamaguchi, 1934) }\end{array}$ & syntype & $\begin{array}{l}\text { Ezo- } \\
\text { zariganimimizu }\end{array}$ \\
\hline 6.44 & ICHUM-1807 & $c-9$ & WM 1 & $\begin{array}{l}\text { Cirrodrilus ezoensis } \\
\text { (Yamaguchi, 1934) }\end{array}$ & syntype & $\begin{array}{l}\text { Ezo- } \\
\text { zariganimimizu }\end{array}$ \\
\hline$"$ & ICHUM-1808 & $"$ & WM 2 & unidentified & & - \\
\hline
\end{tabular}

...Continued on the next page 
TABLE 1. (Continued)

\begin{tabular}{|c|c|c|c|c|c|c|}
\hline $\begin{array}{l}\text { Box / } \\
\text { Slide } \\
\text { No. }\end{array}$ & Catalog No. & Slide Markings & $\begin{array}{l}\text { Specimen(s) } \\
\text { Status }\end{array}$ & Species Ident. & Type Status & $\begin{array}{l}\text { Japanese } \\
\text { common name }\end{array}$ \\
\hline$"$ & ICHUM-1809 & $"$ & WM 3 & $\begin{array}{l}\text { Cirrodrilus ezoensis } \\
\text { (Yamaguchi, 1934) }\end{array}$ & syntype & $\begin{array}{l}\text { Ezo- } \\
\text { zariganimimizu }\end{array}$ \\
\hline$"$ & ICHUM-1810 & $"$ & WM 4 & unidentified & & - \\
\hline $6.46 *$ & ICHUM-1811 & $\begin{array}{l}\text { c.- 4/c.d.s /c.d.1./ } \\
\text { Soranuma }\end{array}$ & WM 1 & $\begin{array}{l}\text { Cirrodrilus ezoensis } \\
\text { (Yamaguchi, 1934) }\end{array}$ & syntype & $\begin{array}{l}\text { Ezo- } \\
\text { zariganimimizu }\end{array}$ \\
\hline$"$ & ICHUM-1812 & $"$ & WM 2 & $\begin{array}{l}\text { Cirrodrilus } \\
\text { megalodentatus } \\
\text { (Yamaguchi, 1934) }\end{array}$ & syntype & $\begin{array}{l}\text { Ooago- } \\
\text { zariganimimizu }\end{array}$ \\
\hline 6.47 & ICHUM-1813 & c. -6 & WM 1 & unidentified & & - \\
\hline$"$ & ICHUM-1814 & $"$ & WM 2 & $\begin{array}{l}\text { Cirrodrilus } \\
\text { megalodentatus } \\
\text { (Yamaguchi, 1934) }\end{array}$ & syntype & $\begin{array}{l}\text { Ooago- } \\
\text { zariganimimizu }\end{array}$ \\
\hline$"$ & ICHUM-1815 & $"$ & WM 3 & $\begin{array}{l}\text { Cirrodrilus ezoensis } \\
\text { (Yamaguchi, 1934) }\end{array}$ & syntype & $\begin{array}{l}\text { Ezo- } \\
\text { zariganimimizu }\end{array}$ \\
\hline$"$ & ICHUM-1816 & $"$ & WM 4 & unidentified & & - \\
\hline$"$ & ICHUM-1817 & $"$ & WM 5 & $\begin{array}{l}\text { Cirrodrilus } \\
\text { megalodentatus } \\
\text { (Yamaguchi, 1934) }\end{array}$ & syntype & $\begin{array}{l}\text { Ooago- } \\
\text { zariganimimizu }\end{array}$ \\
\hline$"$ & ICHUM-1818 & $"$ & WM 6 & unidentified & & - \\
\hline 6.48 & ICHUM-1819 & c. -5 & WM 1 & unidentified & & - \\
\hline$"$ & ICHUM-1820 & $"$ & WM 2 & unidentified & & - \\
\hline$"$ & ICHUM-1821 & $"$ & WM 3 & $\begin{array}{l}\text { Cirrodrilus ezoensis } \\
\text { (Yamaguchi, 1934) }\end{array}$ & syntype & $\begin{array}{l}\text { Ezo- } \\
\text { zariganimimizu }\end{array}$ \\
\hline$"$ & ICHUM-1822 & $"$ & WM 4 & unidentified & & - \\
\hline$"$ & ICHUM-1823 & $"$ & WM 5 & unidentified & & - \\
\hline$"$ & ICHUM-1824 & $"$ & WM 6 & unidentified & & - \\
\hline 6.50 & ICHUM-1825 & c. - 3 / Soranuma & WM 1 & $\begin{array}{l}\text { Cirrodrilus } \\
\text { megalodentatus } \\
\text { (Yamaguchi, 1934) }\end{array}$ & syntype & $\begin{array}{l}\text { Ooago- } \\
\text { zariganimimizu }\end{array}$ \\
\hline$"$ & ICHUM-1826 & $"$ & WM 2 & unidentified & & - \\
\hline$"$ & ICHUM-1827 & $"$ & WM 3 & unidentified & & - \\
\hline$"$ & ICHUM-1828 & $"$ & WM 4 & unidentified & & - \\
\hline 6.51 & ICHUM-1829 & c.m.b / 9 & sect. & - & & - \\
\hline 6.52 & ICHUM-1830 & c.m.b. - 3 / A - $1 * *$ & sect. & - & & - \\
\hline
\end{tabular}

...Continued on the next page 
TABLE 1. (Continued)

\begin{tabular}{|c|c|c|c|c|c|c|}
\hline $\begin{array}{l}\text { Box / } \\
\text { Slide } \\
\text { No. }\end{array}$ & Catalog No. & Slide Markings & $\begin{array}{l}\text { Specimen(s) } \\
\text { Status }\end{array}$ & Species Ident. & Type Status & $\begin{array}{l}\text { Japanese } \\
\text { common name }\end{array}$ \\
\hline 6.53 & ICHUM-1831 & $\mathrm{cmb}-3 / \mathrm{A}-2 * *$ & sect. & - & & - \\
\hline 6.54 & ICHUM-1832 & $\mathrm{cm} b-4 / \mathrm{B}-1 * *$ & sect. & - & & - \\
\hline 6.55 & ICHUM-1833 & c.m.b - 4 / B - $2 * *$ & sect. & - & & - \\
\hline 6.56 & ICHUM-1834 & $\mathrm{c} \mathrm{m} \mathrm{b.} / 6^{* *}$ & sect. & - & & - \\
\hline 6.57 & ICHUM-1835 & $\mathrm{cm} \mathrm{b} / 7 / 7 * *$ & sect. & - & & - \\
\hline $6.58 *$ & ICHUM-1836 & $\mathrm{c} \mathrm{m} \mathrm{b} 2 * *$ & - & original slide & & - \\
\hline 6.59 & ICHUM-1837 & $\begin{array}{l}\text { Box } 6 \text {, Slide } 58 \text { A / } \\
\text { cmb } 2\end{array}$ & WM & $\begin{array}{l}\text { Cirrodrilus } \\
\text { homodontus } \\
\text { (Yamaguchi, 1932) }\end{array}$ & syntype? & $\begin{array}{l}\text { Hoso- } \\
\text { zariganimimizu }\end{array}$ \\
\hline 6.60 & ICHUM-1838 & $\begin{array}{l}\text { Box } 6 \text {, Slide } 58 \text { B / } \\
\text { cmb } 2\end{array}$ & WM & $\begin{array}{l}\text { Cirrodrilus } \\
\text { homodontus } \\
\text { (Yamaguchi, 1932) }\end{array}$ & syntype? & $\begin{array}{l}\text { Hoso- } \\
\text { zariganimimizu }\end{array}$ \\
\hline 6.61 & ICHUM-1839 & $\begin{array}{l}\text { Box } 6 \text {, Slide } 58 \mathrm{C} / \\
\text { cmb } 2\end{array}$ & WM & $\begin{array}{l}\text { Cirrodrilus } \\
\text { homodontus } \\
\text { (Yamaguchi, 1932) }\end{array}$ & syntype? & $\begin{array}{l}\text { Hoso- } \\
\text { zariganimimizu }\end{array}$ \\
\hline 6.62 & ICHUM-1840 & $\begin{array}{l}\text { Box } 6 \text {, Slide } 58 \text { D / } \\
\text { cmb } 2\end{array}$ & WM & $\begin{array}{l}\text { Cirrodrilus } \\
\text { homodontus } \\
\text { (Yamaguchi, 1932) }\end{array}$ & syntype? & $\begin{array}{l}\text { Hoso- } \\
\text { zariganimimizu }\end{array}$ \\
\hline 6.63 & ICHUM-1841 & c.m.b / 8 / 8 & sect. & - & & - \\
\hline 6.64 & ICHUM-1842 & c.m.b. / 12 / c & WM & $\begin{array}{l}\text { Cirrodrilus } \\
\text { homodontus } \\
\text { (Yamaguchi, 1932) }\end{array}$ & syntype? & $\begin{array}{l}\text { Hoso- } \\
\text { zariganimimizu }\end{array}$ \\
\hline 6.65 & ICHUM-1843 & $\mathrm{c} \mathrm{m} \mathrm{b/} 13$ & WM 1 & unidentified & & - \\
\hline$"$ & ICHUM-1844 & $"$ & WM 2 & unidentified & & - \\
\hline$"$ & ICHUM-1845 & $"$ & WM 3 & $\begin{array}{l}\text { Cirrodrilus } \\
\text { homodontus } \\
\text { (Yamaguchi, 1932) }\end{array}$ & syntype? & $\begin{array}{l}\text { Hoso- } \\
\text { zariganimimizu }\end{array}$ \\
\hline$"$ & ICHUM-1846 & $"$ & WM 4 & $\begin{array}{l}\text { Cirrodrilus } \\
\text { homodontus } \\
\text { (Yamaguchi, 1932) }\end{array}$ & syntype? & $\begin{array}{l}\text { Hoso- } \\
\text { zariganimimizu }\end{array}$ \\
\hline 6.67 & ICHUM-1847 & c m b. / 14 & WM & unidentified & & - \\
\hline 6.68 & ICHUM-1848 & $\mathrm{c} \mathrm{m} \mathrm{b} / 15$ & WM & unidentified & & - \\
\hline 6.69 & ICHUM-1849 & $\mathrm{c} \mathrm{m} \mathrm{b/16/c} \mathrm{m} \mathrm{b/9}$ & sect. & - & & - \\
\hline 6.74 & ICHUM-1850 & $\mathrm{c} \mathrm{m} \mathrm{b/} 17 / 4$ & sect. & - & & - \\
\hline
\end{tabular}


TABLE 1. (Continued)

\begin{tabular}{|c|c|c|c|c|c|c|}
\hline $\begin{array}{l}\text { Box / } \\
\text { Slide } \\
\text { No. }\end{array}$ & Catalog No. & Slide Markings & $\begin{array}{l}\text { Specimen(s) } \\
\text { Status }\end{array}$ & Species Ident. & Type Status & $\begin{array}{l}\text { Japanese } \\
\text { common name }\end{array}$ \\
\hline 6.80 & ICHUM-1851 & 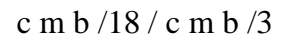 & sect. & - & & - \\
\hline 6.83 & ICHUM-1852 & $13-1$ & sect. & - & & - \\
\hline 6.85 & ICHUM-1853 & $13-2$ & sect. & - & & - \\
\hline 6.87 & ICHUM-1854 & $\mathrm{c} \mathrm{m} \mathrm{b} \mathrm{/} 6-2$ & sect. & - & & - \\
\hline 6.88 & ICHUM-1855 & $6-2$ & sect. & - & & - \\
\hline 6.89 & ICHUM-1856 & $\mathrm{c} \mathrm{m} \mathrm{b/} 13-3$ & sect. & - & & - \\
\hline 6.90 & ICHUM-1857 & 90 & sect. & - & & - \\
\hline 6.91 & ICHUM-1858 & 91 & sect. & - & & - \\
\hline 6.92 & ICHUM-1859 & c.d 1. $10-3$ & sect. & - & & - \\
\hline 6.93 & ICHUM-1860 & c.d 1. $10-2$ & sect. & - & & - \\
\hline 6.94 & ICHUM-1861 & c.d 1. $10-1$ & sect. & - & & - \\
\hline 6.95 & ICHUM-1862 & c.d.1. 9 - 2 & sect. & - & & - \\
\hline 6.96 & ICHUM-1863 & c.d $19-1 / 1$ & sect. & - & & - \\
\hline 6.98 & ICHUM-1864 & $\begin{array}{l}\text { c.d } 1.8 \text { /Sp. c.d.l. / } \\
\text { D } 2\end{array}$ & WM & $\begin{array}{l}\text { Cirrodrilus } \\
\text { megalodentatus } \\
\text { (Yamaguchi, 1934) }\end{array}$ & syntype & $\begin{array}{l}\text { Ooago- } \\
\text { zariganimimizu }\end{array}$ \\
\hline 6.99 & ICHUM-1865 & $\begin{array}{l}\text { c.d.1./ } 7 \text { /Sp. c d 1 /D } \\
3\end{array}$ & WM & $\begin{array}{l}\text { Cirrodrilus } \\
\text { megalodentatus } \\
\text { (Yamaguchi, 1934) }\end{array}$ & syntype & $\begin{array}{l}\text { Ooago- } \\
\text { zariganimimizu }\end{array}$ \\
\hline- & - & $100 /$ A 3 & sect. & - & & - \\
\hline- & - & $101 / \mathrm{A} 4$ & sect. & - & & - \\
\hline- & - & 103 / A6-1 & sect. & - & & - \\
\hline- & - & $106 /$ A A7 & WM & $\begin{array}{l}\text { Cirrodrilus cirratus } \\
\text { Pierantoni, } 1905\end{array}$ & non-type & $\begin{array}{l}\text { Kamuri- } \\
\text { zariganimimizu }\end{array}$ \\
\hline- & - & 108 & WM & $\begin{array}{l}\text { Cirrodrilus cirratus } \\
\text { Pierantoni, } 1905\end{array}$ & non-type & $\begin{array}{l}\text { Kamuri- } \\
\text { zariganimimizu }\end{array}$ \\
\hline- & - & 113 / A16 & WM & $\begin{array}{l}\text { Cirrodrilus cirratus } \\
\text { Pierantoni, } 1905\end{array}$ & non-type & $\begin{array}{l}\text { Kamuri- } \\
\text { zariganimimizu }\end{array}$ \\
\hline- & - & 114 / A' 37-1A' 37-2 & sect. & - & & - \\
\hline- & - & $119 / 32-2$ & sect. & $\begin{array}{l}\text { Cirrodrilus cirratus } \\
\text { Pierantoni, } 1905\end{array}$ & non-type & $\begin{array}{l}\text { Kamuri- } \\
\text { zariganimimizu }\end{array}$ \\
\hline
\end{tabular}


TABLE 1. (Continued)

\begin{tabular}{|c|c|c|c|c|c|c|}
\hline $\begin{array}{l}\text { Box / } \\
\text { Slide } \\
\text { No. }\end{array}$ & Catalog No. & Slide Markings & $\begin{array}{l}\text { Specimen(s) } \\
\text { Status }\end{array}$ & Species Ident. & Type Status & $\begin{array}{l}\text { Japanese } \\
\text { common name }\end{array}$ \\
\hline- & - & 120 & WM & $\begin{array}{l}\text { Cirrodrilus cirratus } \\
\text { Pierantoni, } 1905\end{array}$ & non-type & $\begin{array}{l}\text { Kamuri- } \\
\text { zariganimimizu }\end{array}$ \\
\hline- & - & 123 & sect. & - & & - \\
\hline- & - & $124 /$ Tot E & WM & $\begin{array}{l}\text { Cirrodrilus } \\
\text { nipponicus } \\
\text { (Yamaguchi, 1932) }\end{array}$ & syntype & $\begin{array}{l}\text { Nippon- } \\
\text { zariganimimizu }\end{array}$ \\
\hline- & - & 125 / E18 & WM & $\begin{array}{l}\text { Cirrodrilus } \\
\text { nipponicus } \\
\text { (Yamaguchi, 1932) }\end{array}$ & syntype & $\begin{array}{l}\text { Nippon- } \\
\text { zariganimimizu }\end{array}$ \\
\hline- & - & $131 /$ F10 & sect. & - & & - \\
\hline- & - & 133 / F12 & sect. & - & & - \\
\hline
\end{tabular}

Abbreviations according to column: * in Box / Slide No. column, specimen was remounted.

Catalog No. ICHAM, Invertebrate Collection of the Hokkaido University Museum, Sapporo, Japan, previously the Zoological Institute, Hokkaido University (ZIHU); USNM, National Museum of Natural History, Smithsonian Institution, Washington D.C., U.S.A.

** in Slide Markings column, Japanese characters present on slide; WM / sect., specimens whole mounted (WM) or sectioned (sect.); ?, tentative identification or "unidentified" species with specimens; -, no sections were identified.

\section{Slide collection organization and examination}

As already noted, Prof. Yamaguchi's records and slide catalog have not been found to date, but there is no evidence to indicate they have been destroyed. Therefore it is vital that the slides be kept in the original order in their boxes so that details in the catalog, when found, can be unambiguously assigned to a slide. Therefore, each slide was given a reference number based on its slide box and slot number; as an example, the slide in box 2, slot 46, is referred to as 2.46 (Table 1). There is no information about the slides on the box lids, but many slides carry abbreviations in Roman letters and Arabic numbers (Table 1, Slide Markings), plus occasional names or characters; the latter being indicated by ** in Table 1. Although some of these letters have an obvious interpretation, e.g., "c.s." being cross section (corroborated by viewing the sections), other letters and numbers are meaningless without the catalog.

Prior to depositing the collection, Prof. Ohtaka arranged for slides to be assigned sequential catalog numbers from the museum in the Zoological Institute, Hokkaido University, Japan, using the prefix ZIHU. Subsequently, the museum had a name change and it is now called the Invertebrate Collection of the Hokkaido University Museum, Sapporo, Japan, and so holdings now have the prefix ICHUM although catalog numbers remain the same. It is important to clearly state this change as the slides referred to in Gelder \& Ohtaka (2002) carry the old ZIHU prefix, while their ICHUM designation is used in this article.

As the morphological characters of the jaws and peristomial appendages in the serial, stained sections could not be easily recognized during the preliminary study, no attempt was made to identify the species. While the sections would not allow the jaws to be reconstructed, the arrangement of peristomial tentacles and other surficial features can be determined-including the taxonomically important positions of the anterior nephidial pores and the male reproductive organs. As these organs are important features in determining a species in many branchiobdellidans, identification of this material is probable. However, the male system has only been described in detail in recent new species from Japan, although Yamaguchi (1934: Fig. 4) did provide such information for Cirrodrilus inukaii and Gelder (1987) for C. cirratus and C. uchidai. Therefore, for the male reproductive system to be fully utilized in an identification, the morphology needs to be described in newly collected specimens of the remaining Japanese species. 
The whole mounted specimens were examined with a Nikon research microscope using bright-field, darkfield, phase contrast, and differential interference contrast (DIC) illuminations. Slides with a broken coverglass, or with air-bubbles touching a specimen were remounted and examined.

Whole-mounted specimens were identified and the name of the species was written on the slide label; if the identification was equivocal, a question mark was added. Some slides had multiple specimens on them and so each specimen was assigned a number, written on the coverglass in ink. As an example, slide 6.43 (ICHUM-1797) had seven specimens on it; these are designated WM 1 to WM 7 becoming (ICHUM-1797 to ICHUM-1806) with an identification given for each, which were indicated both on the slide label and in Table 1 . Where the mounting medium was damaged the specimens were remounted and this is indicated by an asterisk (*) in Table 1. In one case, the four specimens on slide 6.58 (ICHUM-1836) were remounted on new, separate slides and cataloged as 6.59 to 6.62 (ICHUM-1837 to ICHUM-1840), and the original slide (6.58) with its written characters was returned to its original slot. Six slides (box/slot nos. 2.82, 2.97, 3.26, 3.67, $4.48,4.76)$ had either been broken into many pieces and/or the specimens had cracked into small pieces, and were beyond restoration. These destroyed slides were not returned to the original slide box slots, but they were assigned museum catalog numbers so their fate could be traced when the original laboratory records are found.

In 2018, ten slides with branchiobdellidans were found scattered among Prof. Yamaguchi's oligochaete collection in Hakodate University. The specimens were in poor condition, and have not been entered into the museum's register, but placed at the end of Table 1 for completeness.

\section{Results and Discussion}

The rediscovered slide collection was found to contain specimens of all 17 species described by Prof. Yamaguchi, together with specimens of three other species described after his death. These latter species included Holtodrilus truncatus (Liang, 1963) (slides 4.14 and 4.15), Branchiobdella macroperistomium Liu \& Zhang, 1983 (slides 5.4 and 5.18), and Branchiobdella monodontus Liu \& Zhang, 1983 (slides 4.8 and 4.9). Yamaguchi (1934:177, 178) obtained specimens of the European Branchiobdella pentadonta Whitman, 1882, from Prof. W. Michaelsen of Hamburg, Germany, for comparison with his Oriental material. Whole mounts on slides 5.1 (ICHUM-1683), 5.2 (ICHUM-1684), 5.3 (ICHUM-1685), 5.73 (ICHUM-1728) carry the name B. pentadonta (Table 1). It is probable that some of the other specimens supplied by Michaelson were sectioned by Yamaguchi and placed in the collection. Such material could be identified in the future as the male reproductive organs and number of paired testes in Branchiobdella differs from those in Cirrodrilus.

The type description of Cirrodrilus cirratus Pierantoni, 1905 was based on poorly preserved and deformed specimens; the type having its body rotated $180^{\circ}$ in relation to its head, hence dorsal appendages were called "ventral cirri" (Pierantoni 1905: fig. 1). Yamaguchi (1932a) made a detailed redescription of $C$. cirratus, and as a result was able to recognize a different but very similar species, Cirrodrilus uchidai (Yamaguchi, 1932). Although he studied their reproductive systems, little information was included in his paper. Subsequent studies of $C$. uchidai specimens showed that some morphological characters-body length, peristomial tentacle length and the presence and number of dorsal segmental appendages - were quite variable (Yamaguchi 1934: 212, fig. 15 A, B, C, and D). Whether such variablity is intraspecific or an indication of a species complex is unknown; however, the specimens examined by Yamaguchi-and recently by us as discussed in this paper-are cataloged in the ICHUM collection for future investigation.

Pierantoni (1906) described a second Japanese species, but because it lacked segmental "cirri" he placed it in a new genus, Stephanodrilus. He (Pierantoni 1912) then described another two species and placed them in the same genus. In subsequent works these species were assigned to various genera, but Yamaguchi (1934) reverted back to using Stephanodrilus (Gelder \& Ohtaka 2002). Professor Yamaguchi gave a few of his specimens to Prof. P.C. Holt, and this allowed Holt (1967: 2-3), using the Principle of Priority as presented in Art. 23.1 of the International Code of Zoological Nomenclature (International Commission on Zoological Nomenclature 2000), to state that Cirrodrilus has historical priority and is therefore the correct name for the genus.

The jaws provide a major diagnostic character for Cirrodrilus nipponicus (Yamaguchi, 1932c). The ends of the ridge supporting the small teeth on the anterior-facing surface of the jaws curve inwards toward their respective bases. This curling of the lateral ends is not always easy to distinguish, but it is clearly shown in slides 1.4 (ICHUM-1399), 1.86 (ICHUM-1463), and 1.88 (ICHUM-1464). 
Yamaguchi (1934: 198, 199) recognized that only three character states separate Cirrodrilus ezoensis (Yamaguchi, 1934) from Cirrodrilus megalodentatus (Yamaguchi, 1934), as the two species are identical in all other respects. These characters are: the position of the anterior nephridiopores on segment 3 , and the difference in size and number of small teeth on the dorsal jaws. In the specimens available, the position of the anterior nephridiopores could not be determined. The number of small teeth on the dorsal jaw of C. ezoensis is eight and for C. megalodentatus is six (Yamaguchi 1934:197, 198); however, these numbers were observed to vary by plus or minus two. The difference in size of the dorsal jaw in the two species is significant, and this was verified by measuring the dimensions of the dorsal jaws from both species. The dorsal jaw in ten specimens of $C$. ezoensis was measured (width of jaw base, 15.0 to $15.2 \mathrm{~mm}$; height of median tooth from base to apex, 15.8 to $20.5 \mathrm{~mm}$ ) and compared to measurements made on 11 specimens of $C$. megalodentatus (width of jaw base, 25.3 to $30.0 \mathrm{~m}$; height of median tooth from base to apex, 26.8 to $31.6 \mathrm{~m}$ ). When the jaws are in a direct dorso-ventral orientation, the sides of the central, dorsal tooth appear straight in C. ezoensis but convex in C. megalodentatus. Although shown by Yamaguchi (1934:197, fig.7B, and 198, fig. 8B), this feature was not easy to recognize until the syntype specimens were found for reference (Table 1).

Two specimens of Hidejiodrilus koreanus (Pierantoni, 1912) were identified (slides 1.46 (ICHUM-1431) and 1.99 (ICHUM-1466) based on the characteristic appearance of the jaws, with all of them having a 3-1-3/ 3-1-3 dental formula. This is an important discovery as the type material no longer exists in the Hamburg Museum, Germany (Gelder 1987). Gelder \& Brinkhurst (1990) recognized this species was not a member of the Cirrodrilus and transferred it to the new monotypic Hidejiodrilus based on the type description. As this did not include any details of the male reproductive system, the new genus could not be assigned to a family and was designated incertae sedis by Gelder (1996). After receiving specimens from Korea, Gelder (2010) redescribed the species, including the male reproductive system, which allowed the genus to be placed in the subfamily Bdellodrilinae. The examined specimens were then designated as a neotype and two neoparatypes.

Signal crayfish, Pacifastacus leniusculus (Dana, 1852), from Oregon, U.S.A., were introduced into Japan five times between 1926 to 1930 (Kamita 1970; Kawai 2017), and they carried branchiobdellidans which were named, Cambarincola okadai Yamaguchi, 1933. The type description only included body shape and jaw structure, and these were similar to the subsequently described Triannulata montanus Goodnight, 1940 from Washington State, USA. Holt (1974) recognized the latter species had been placed incorrectly in the Triannulata, and transferred it to Cambarincola montanus (Goodnight, 1940). By this time the Yamaguchi collection was presumed lost, and so Holt and Opell (1993: 253) considered C. okadai to be nomen inquirendum. During the examination of the rediscovered collection, four specimens of $C$. okadai were identified, including the individual drawn in Yamaguchi's (1933: fig. 1) type description. This resulted in a redescription of the species, the names Triannulata montanus and Cambarincola montanus reduced to junior synonyms, and the four specimens being designated as syntypes (Gelder \& Ohtaka 2000b). Three of these specimens were deposited in the Invertebrate Collection of the Hokkaido University Museum, Sapporo (ICHAM) - slide 2.20 is designated the lectotype (ICHUM-1347) and slides 2.4 and 2.10 become paralectotypes (ICHUM-1348, ICHUM-1349). The fourth specimen-a third paralectotype, slide 2.9 (USNM 186575)—was placed in the National Museum of Natural History-Smithsonian Institution, Washington D.C., U.S.A.

Information from a series of taxonomical studies by Yamaguchi, plus the history of Prof. Yamaguchi's research (Mikami 1963) and an interview with Prof. Kamihira by Prof. Ohtaka, has enabled the status of some identified specimens to be determined. Yamaguchi's (1934) collection and descriptions of six species, Cirrodrilus inukaii, C. aomorensis, C. ezoensis, C. megalodentatus, C. makinoi and C. chosen, are only found in that publication, hence these specimens were designated syntypes. In contrast, additional specimens were used to supplement the original descriptions for three Japanese species (Cirrodrilus uchidai, C. homodontus and C. nipponicus) and four mainland species (Branchiobdella orientalis, B. kobayashii, C. kawamurai and C. suzukii), therefore these slides in the collection probably contain both original (syntype) and additional (nontype) specimens; hence they are labeled as "syntype ?" in Table 1.

The Yamaguchi slide collection provides an invaluable resource for future research on East Asian branchiobdellidans, and this review of current information will greatly assist researchers, particularly those investigating the sectioned material in the collection. 


\section{Acknowledgments}

The authors wish to thank emeritus Prof. Yukiyoshi Kamihira, Hakodate Unversity, Hakodate, for the loan of the Prof. H. Yamaguchi branchiobdellidan slide collection, emeritus Prof. Haruo Katakura, Hokkaido Unversity, for his help in depositing the collection in the permanent museum archives of the Invertebrate Collection of the Hokkaido University Museum, Sapporo, Japan, and Dr. Andrea M. Gorman, University of Maine at Presque Isle, U.S.A. for her assistance. Support for this project came from the Student Work-Study Program, Faculty Development Mini-grants and Sabbatical Support Funds from the University of Maine at Presque Isle, and a research grant-in-aid in support of aquaculture from the University of Maine at Orono, U.S.A. to S.R.G. The authors also wish to thank Steven V. Fend, Mark J. Wetzel and an anonymous reviewer for their constructive criticisms of the manuscript.

\section{References}

Ahn, D.-H. \& Min, G.-S. (2016) First report of the branchiobdellidan Holtodrilus truncatus (Annelida: Clitellata) found on the freshwater atyid shrimp Neocaridina sp. from Korea. Journal of Species Research, 5, 459-462. https://doi.org/10.12651/JSR.2016.5.3.459

Bănărescu, P. (1990) Zoogeography of Fresh Waters. Aula-Verlag Wiesbaden, Germany, 1617 pp.

Brinkhurst, R.O. \& Gelder, S.R. (1991) Annelida: Oligochaeta and Branchiobdellida. In Thorp, J.H. \& Covich F. (Eds.), Ecology and Classification of North American Freshwater Invertebrates. Academic Press, New York, pp. 401-435.

Fujita, Y., Kawahara, T., Niwa, N. \& Shokita, S. (2010) First record of Holtodrilus truncatus (Liang, 1963) (Annelida: Clitellata: Branchiobdellidae) from the Ryukyu Islands. The Biological Magazine Okinawa, 48, 25-33. [in Japanese with English abstract]

Gelder, S.R. (1987) Observations on three species of branchiobdellid (Annelida: Clitellata) worms from eastern Asia. Hydrobiologia, 155, 15-25. https://doi.org/10.1007/BF00025627

Gelder, S.R. (1996) A review of the taxonomic nomenclature and a checklist of the species of the Branchiobdellae (Annelida: Clitellata). Proceedings of the Biological Society of Washington, 109, 653-663.

Gelder, S.R. (1999) Zoogeography of branchiobdellidans (Annelida) and temnocephalans (Platyhelminthes) ectosymbiotic on freshwater crustaceans, and their reactions to one another in vitro. Hydrobiologia, 406, 21-31. https://doi.org/10.1023/A:1003755630718

Gelder, S.R. (2010) Re-description of the branchiobdellidan, Hidejiodrilus koreanus (Pierantoni, 1912), (Annelida: Clitellata), from the Republic of Korea, and the designation of a neotype and paraneotype specimens. Acta zoologica bulgarica, 62, 21-26.

Gelder, S.R. (2011) Reassignment of a Central American species of the Branchiobdellida (Annelida: Clitellata) to Forbesodrilus n.g. Acta zoologica bulgarica, 63, 119-123.

Gelder, S.R. \& Brinkhurst, R.O. (1990) An assessment of the phylogeny of the Branchiobdellida (Annelida: Clitellata) using PAUP. Canadian Journal of Zoology, 68, 1318-1326. https://doi.org/10.1139/z90-197

Gelder, S.R. \& Ohtaka, A. (2000a) Description of a new species and a redescription of Cirrodrilus aomorensis (Yamaguchi, 1934) with a detailed distribution of the branchiobdellidans (Annelida: Clitellata) in Northern Honshu, Japan. Proceedings of the Biological Society of Washington, 113, 633-643.

Gelder, S.R. \& Ohtaka, A. (2000b) Redescription and designation of lectotypes of the North American Cambarincola okadai Yamaguchi, 1933 (Annelida: Clitellata: Branchiobdellidae). Proceedings of the Biological Society of Washington, 113, 1087-1095.

Gelder, S.R. \& Ohtaka, A. (2002) A review of the oriental branchiobdellidans (Annelida: Clitellata) with reference to the rediscovered slide collection of Prof. Hideji Yamaguchi. Species Diversity, 7, 333-344. https://doi.org/10.12782/specdiv.7.333

Gelder, S.R. \& Williams B.W. (2016) Global Overview of Branchiobdellida (Annelida: Clitellata). In: Kawai, T., Faulkes, Z. \& Scholtz, G. (Eds.), Freshwater Crayfish: A Global Overview. CRC Press, New York, pp. 628-654.

Holt, P.C. (1967) Status of genera Branchiobdella and Stephanodrilus in North America with description of a new genus (Clitellata: Branchiobdellida). Proceedings of the United States National Museum, 124, 1-10. https://doi.org/10.5479/si.00963801.124-3631.1

Holt, P.C. (1974) An emendation of the genus Triannulata Goodnight, 1940, with the assignment of Triannulata montana to Cambrincola Ellis 1912 (Clitellata: Branchiobdellida). Proceedings of the Biological Society of Washington, 87, 57-72. 
Holt, P.C. \& Opell, B.D. (1993) A checklist of and illustrated key to the genera and species of the Central and North American Cambarincolidae (Clitellata: Branchiobdellida). Proceedings of the Biological Society of Washington, 106, 251-295.

International Commission on Zoological Nomenclature (2000) International Code of Zoological Nomenclature. Available from: http://www.nhm.ac.uk/hosted-sites/iczn/code/ (accessed 4 October 2018)

Kamita, T. (1970) Crayfishes brought to Japan from Mexico. Zoological Magazine (Tokyo), 82, 123-126 [In Japanese]

Kawai, T. (2017) Observation on mandible and gill morphology in Pacifastacus leniusculus and Cherax quadricarinatus with a review of the introduction of alien crayfish into Japan. Freshwater Crayfish, 23, 29-39.

Kitayabu, J., Ohtaka, A. \& Yamato, S. (2016) Symbiotic ecology of Holtodrilus truncatus (Annelida, Clitellata, Branchiobdellida) in the southern Kii Peninsula, Japan. Nanki-Seibutsu, 58, 190-196. [in Japanese]

Liang, Y.-L. (1963) Studies on the aquatic Oligochaeta of China. I. Descriptions of new naids and branchiobdellids. Acta Zoologica Sinica, 15, 560-570.

Liu, S.-C. (1964) A second report of Branchiobdellidae in Liaoning Province with descriptions of three new species. Acta Zoologica Sinica, 16, 602-610.

Liu, S.-C. (1984) Descriptions of two new species of the genus Stephanodrilus from Northeast China and notes on St. trunctus Liang from Guagdong Province (Oligochaeta: Branchiobdellidae). Acta Zootaxonomica Sinica, 9, 351-355.

Liu, S.-C. \& Chang, C.-A. (1964) On four new species of Branchiobdellidae from crayfish, Cambaroides dauricus (Pallas). Acta Zoologica Sinica, 16, 33-38.

Liu, S.-C. \& Zhang, D.-C. (1983) Three new species of the genus Branchiobdella (Oligochaeta: Branchiobdellidae) from China. Acta Zootaxonomica Sinica, 8, 246-251.

Mikami, T. (1963) Resume and author bibliography of Prof. Hideji Yamaguchi. Seibutsu-Kyouzai no Kaitaku, Hokkaido University of Education, 3, iii-vi. [in Japanese]

Niwa, N., Ohtomi, J., Ohtaka, A. \& Gelder, S.R. (2005) The first record of the ectosymbiotic branchiobdellidan Holtodrilus truncatus (Annelida, Clitellata) and on the fresh-water shrimp Neocaridina denticulata denticulata (Caridea, Atyidae) in Japan. Fisheries Science, 71, 685-687. https://doi.org/10.1111/j.1444-2906.2005.01017.x

Ohtaka, A. (2010) Community conservation. In: Kawai, T. \& Takahata, M. (Eds.), Biology of Crayfish. Hokkaido Daigaku Shuppankai, Sapporo, pp. 445-475. [In Japanese]

Ohtaka, A. \& Chen, R.-T. (2010) New records of a branchiobdellidan and four microdrile oligochaetes (Annelida: Clitellata) from inland waters of Taiwan. Taiwan Journal of Biodiversity, 12, 97-110.

Ohtaka, A. \& Gelder, S.R. (2015) Description of a new species of Branchiobdellida (Annelida: Clitellata) and comparison with other Cirrodrilus species in northern Honshu, Japan. Species Diversity, 20, 67-71. https://doi.org/10.12782/sd.20.1.067

Ohtaka, A., Gelder, S.R., Nishino, M., Ikeda, M., Toyama, H., Cui Y.-D., He, X.-B., Wang, H.-Z., Chen, R.-B. \& Wang, Z.-Y. (2012) Distributions of two ectosymbionts, branchiobdellidans (Annelida: Clitellata) and scutariellids (Platyhelminthes: "Turbellaria": Temnocephalida), on atyid shrimp (Arthropoda: Crustacea) in southeast China. Journal of Natural History, $46,1547-1556$. https://doi.org/10.1080/00222933.2012.692826

Pierantoni, U. (1905) Cirrodrilus cirratus n.g. n.sp. parassita dell' Astacus japonicis. Annuario del Museo Zoologico della R. Università di Napoli, 1, 1-3.

Pierantoni, U. (1906) Nuovi Discodrilidi del Giappone e della California. Annuario R. Museo zoologico della R. Università di Napoli, 2, 1-9.

Pierantoni, U. (1912) Monografia dei Discodrilidae. Annuario del Museo Zoologico della R. Università di Napoli (new series), $3,1-27$.

Subchev, M.A. (1986) On the Korean branchiobdellids (Annelida: Clitellata) with a description of a new species Branchiobdella teresae sp.n. Acta Zoologica Bulgarica, 31, 60-66.

Subchev, M.A., Stanimirova, L.S. \& Tomek, T. (1991) Distribution of branchiobdellidans (Annelida, Clitellata) on the Korean Peninsula. Acta Zoologica Bulgarica, 41, 12-17.

Tanaka, K., Wada, K. \& Hamasaki, K. (2016) Distribution of Holtodrilus truncatus, a branchiobdellidan ectosymbiotic on atyid shrimps in the Kii peninsula, western Japan, with reference to salinity tolerance and host preference. Zoological Science, 33, 154-161. https://doi.org/10.2108/zs150049

Timm, T. (1991) Branchiobdellida (Oligochaeta) from the farthest South-East of the U.S.S.R. Zoologica Scripta, 20, 321-331. https://doi.org/10.1111/j.1463-6409.1991.tb00297.x

Timm, T. (1999) Distribution of freshwater oligochaetes in the west and east coastal regions of the North Pacific Ocean. Hydrobiologia, 406, 67-81. https://doi.org/10.1023/A:1003749519662 
Williams, B.W., Gelder, S.R., Proctor, H. \& Coltman, D.W. (2013) Molecular phylogeny of North American Branchiobdellida (Annelida: Clitellata). Molecular Phylogenetics and Evolution, 66, 30-42.

https://doi.org/10.1016/j.ympev.2012.09.002

Yamaguchi, H. (1932a) On the genus Cirrodrilus Pierantoni, 1905 with a description of a new branchiobdellid from Japan. Annotationes Zoologicae Japonenese, 13, 361-367.

Yamaguchi, H. (1932b) A new species of Cambarincola, with remarks on spermatic vesicles of some branchiobdellid worms. Proceedings of the Imperial Academy, Japan, 8, 454-456. https://doi.org/10.2183/pjab1912.8.454

Yamaguchi, H. (1932c) Description of a new branchiobdellid, Carcinodrilus nipponicus n.g. et n.sp. Journal of the Faculty of Science, Hokkaido University, series VI, Zoology, 2, 61-67.

Yamaguchi, H. (1933) Description of a new branchiobdellid, Cambarincola okadai n.sp., parasitic on American crayfish transferred into a Japanese lake. Proceedings of the Imperial Academy, Japan, 9, 191-193. https://doi.org/10.2183/pjab1912.9.191

Yamaguchi, H. (1934) Studies on Japanese Branchiobdellidae with some revisions on the classification. Journal of the Faculty of Science, Hokkaido University, series VI, Zoology, 3, 177-219.

Yamaguchi, H. (1935a) Family Branchiobdellidae (Class Clitellata). Fauna Nipponica, vol. 6. (3). Sanseido Publishing Co., Tokyo, 37 pp. [in Japanese]

Yamaguchi, H. (1935b) On the Branchiobdellidae, a special group of Oligochaeta. Botany and Zoology, Tokyo, 3, 552-560. [in Japanese]

Yamaguchi, H. (1940) Oligochaeta of Manchoukuo. In: Report of the limnological survey of Kwantung and Manchoukuo, pp. 382-394. [in Japanese]

Yamaguchi, H. (1954) A catalogue of the aquatic Oligochaeta in Japan. Bulletin of the Hokkaido Gakugei Daigaku, 5, 93-120. [in Japanese] 